\title{
Über die neue Tuberkulosewelle; nebst einem Beitrag zum jetzigen Stand der Tonsillartuberkulose.
}

\author{
Von \\ Dr. med. H. Cramer.
}

(Aus dem pathologischen Institut des allgemeinen Krankenhauses Hamburg-Barmbeck. [Prosektor Prof. Dr. Th. Fahr].)

Mit 2 Textabbildungen.

Nachdem wir uns in der schweren Belastungsprobe 1914 bis 1918 als das körperlich und geistig leistungsfähigste und damit gesundeste Volk der Alten Welt erwiesen haben (daran ändert ein vorübergehender Erschöpfungs- und Verwirrungszustand nichts), sind wir meines Erachtens berechtigt zu der Hoffnung, daß der Kampf ums Dasein selbst in der unglaublich scharfen Form, wie wir ihn uns erwachsen sehen, nicht dazu dienen soll, uns dem Untergang entgegenzuführen.

Ich setze diese Ưberzeugung meinen Ausführungen voraus, da dieselben so unerfreuliche Tatsachen von neuem belegen, daß es unseres festen Vertrauens auf unsere Kraft bedürfen wird, wenn wir uns nicht durch Skeptizismus in der erforderlichen intensivsten Aktivität lähmen lassen wollen.

Der gefährlichste Feind unserer Volksgesundheit, die Tuberkulose, die uns bereits im Frieden nach Seiler jeden vierten Menschen im erwerbsfähigen Alter raubte und die wir in hartem Kampfe langer Friedensjahre mit dem mächtigsten Verbündeten, einer ständig fortschreitenden Hebung der äußeren Lebensbedingungen so wirksam bekämpften, daß wir ihn aus der Offensive in die Defensive getrieben hatten, hat nach vollkommen veränderter Lage der Dinge mit erschütternder Gesetzmäßigkeit die Offensive auf allen Fronten von neuem begonnen.

Da wir für den Kampf gegen die Tuberkulose alle verfügbaren mittelbaren und unmittelbaren Hilfskräfte mobilisieren müssen und das Alarmsignal gar nicht eindringlich genug gegeben werden kann, fühle ich mich berechtigt, meine diesbezüglichen Feststellungen niederzulegen, obschon das zugrunde liegende Material nicht ganz so umfangreich ist, wie es für derartige statistische Zwecke wohl wünschenswert wäre.

Wenn hierbei die pathologische Anatomie als Unterlage klinischer Bewertung des Krankheitszustandes eines Individuums den immun-biologischen Untersuchungsmethoden insofern nachsteht, als sie uns nur über Zustandsbilder Auskunft gibt, die vielleicht nicht die allerersten Vorpostengefechte wiedergeben, so dürfte es bis auf weiteres doch noch sehr verfrüht sein, ihr 
den wichtigen Platz in dem Kampf gegen die Tuberkulose bis zu einem gewissen Grade absprechen zu wollen, wie dies von serologischer Seite meines Erachtens sehr zu Unrecht bereits geschieht. Solange der Kampf sich auf dem Boden histologischer Veränderungen abspielt, bleiben diese die Basis zur Beurteilung der Lage der Dinge. Ja, manche Ergebnisse, die, wie die Untersuchungen Bessaus das tuberkulöse Gewebe als Träger der Tuberkulinüberempfindlichkeit-erkannt haben, sind geeignet, den scheinbaren Gegensat\% zu beseitigen und lassen uns hoffen, daß wir auch an Hand histologischer Veränderungen einen ergänzenden Einblick in die sie bedingenden immun-biologischen Vorgänge erhalten werden.

Im Verfolg einer demnächst erscheinenden Arbeit von Herrn Prof. Fahr, in welcher dieser die bedenkliche Zunahme tuberkulöser Prozesse im Kindesalter und andererseits auch im Greisenalter betont, habe ich diese Tatsache an dem Material des Instituts für das Jahr 1919 und das erste Halbjahr 1920 weiter verfolgen können. Ich habe von neuem zum Vergleich die Verbreitung der Tuberkulosefälle aus dem Jahre 1915 herangezogen, die etwa den Friedenverhältnissen entsprechen. Ein Vergleich der Untersuchungsergebnisse anderer zahlreicher diesbezüglicher Feststellungen ist deshalb sehr erschwert, weil die Gesichtspunkte, nach welchen die Untersuchung des Materials erfolgte, nicht einheitlich sind und in der Häufigkeit der Tuberkulose offenbar regionär die größten Differenzen bestehen. Hamburg hatte speziell nach den Statistiken von Seiler vor dem Kriege nächst Wiesbaden und Charlottenburg die niedrigste Tuberkulosemortalität. Wir haben daher zum Vergleich nur die eigenen, unter denselben Bedingungen gewonnenen Resultate herangezogen und unsere Hauptaufgabe darin erblickt, alle aktiven tuberkulösen Prozesse zu erfassen, um einen Eindruck von der Tatsache, Geschwindigkeit und Eigenart des Fortschreitens der Tuberkuloseoffensive zu erhalten, d. h. also, wir haben nicht nur die Tuberkulose-Todesfälle registriert, sondern auch die Fälle, die eine latente aktive Tuberkulose ergaben, also die Fälle, in denen durch interkurrierende andere Todesursachen das Umsichgreifen des tuberkulösen Prozesses abgebrochen wurde (Flügge, ähnlich Kraemer). Andererseits haben wir die inaktive, latente Tuberkulose, $d$. h. ausgeheilte und verkalkte Tuberkuloseherde nicht mit aufgenommen, um nur die wirklichen derzeitigen fortschreitenden Erkrankungen zu fixieren. Hamburger charakterisiert diese Verhältnisse folgendermaßen: „Die Tuberkulose ist ein Prozeß, der sich nur unter bestimmten Bedingungen auch als Krankheit manifestiert,"... . , alle, die sich infizieren, bekommen eine Tuberkulose in streng wissenschaftlichem Sinne, aber nur ein geringer Bruchteil von ihnen erkrankt." Dabei sind wir uns der Tatsache wohl bewußt, daß auch die scheinbar harmlosen, latenten Prozesse in der überwiegenden Mehrzahl lebenslänglich eine Gefahr für ihren Träger bedeuten (Ha mburger), wie auch gerade aus unseren Untersuchungen über Alterstuberkulose hervorgeht, und da 3 selbst Kreideherde in $75 \%$ und steinhartes Material in 35\% nach den Untersuchungen von Lubarsch noch infektiös sind. Aber es handelt sich dann eben um ein Stadium des nie ruhenden Kampfes zwischen Organismus und Infektionserreger, in welchem der erstere offenbar einen siegreichen Frieden geschlossen hat, wenn auch nur einen bcwaffneten Frieden. 
Um möglichst wenig den kleinen Schwankungen durch geringere Zahl der verwerteten Fälle ausgesetzt zu sein, habe ich dieselben in nur vier Unterabteilungen gegliedert, und zwar je für das Alter von: 1-15 Jahren, 15-30 Jahren, 30-60 Jahren und über 60 Jahren; also Kindheit, Jugend, Reife und Alter getrennt. Bei der Beobachtung des Tuberkulosematerials und histologischer Untersuchung der lymphatischen Elemente des Respirationstraktus fiel uns eine abnorm starke Beteiligung der Tonsillen an dem tuberkulösen Prozeß auf, und daher wurden diese systematisch in einer größeren Reihe von Fällen untersucht, worïber späterhin ebenfalls berichtet werden soll.

Kraemer machte darauf aufmerksam, daß bei Verallgemeinerung der Krankenhausstatistiken noch mehr zu beachten sei, daß die Krankenhauspatienten durchweg minderbemittelten Kreisen entstammen, in denen die Tuberkulose weit verbreiteter ist als in den übrigen Volksschichten. Das ist eine Tatsache, die wir auf Schritt und Tritt bestätigt finden und auch wieder in dem instruktiven statistischen Werk Seilers am klarsten und einfachsten festgelegt ist. Seiler fand, daß im Jahre 1910 unter den Steuerzahlern mit höchstens $1200 \mathrm{Mk}$. Einkommen 50,9 von 10000 an 'Tuberkulose starben und sich diese hohe Ziffer genau parallel dem höheren Einkommen verkleinert, so daß bei einem Einkommen von 25000 bis $50000 \mathrm{Mk}$. nur noch 5,5 pro 10000 Todesfälle an Tuberkulose zu verzeichnen sind. Bis zu einem gewissen Grade trifft das natürlich auch jetzt noch $\mathrm{zu}$, nur fürchte ich, hat die Rationierung der Lebensmittel und neuerdings auch die Rationierung der Wohnungen mit dem durch die Kohlennot gegebenen Zwang engsten Zusammenlebens aller Familienmitglieder für die Verbreitung der Tuberkulose einen für das Volkswohl wenig wünschenswerten, weitgreifenden Ausgleich geschaffen, so dals der Klassenunterschied in dieser Beziehung nicht mehr so bedeutend sein wird als vor dem Kriege.

Für das Verständnis der zu schildernden Verhältnisse ist es zweckmäßig, einige allgemein wichtige Gesichtspunkte kurz zu berïhren und ich habe soeben gleich der beiden wichtigsten Ursachen für die Verbreitung der Tuberkulose überhaupt Erwähnung getan. Die direkte Abhängigkeit der'Tuberkuloseverbreitung von der wirtschaftlichen Lage der Bevölkerung bei gleichen klimatischen Verhältnissen und gleichem Durchseuchungszustand ist bedingt durch den Stand der daraus resultierenden hygienischen Lebensmöglichkeiten, wie es der Charakter der Tuberkulose als einer typischen Infektionskrankheit ja auch wahrscheinlich macht. Wie bekannt, wird die Tuberkulose durch die Tröpfcheninfektion übertragen und ihre Weiterverbreitung steht und fällt mit der Enge des Zusammenlebens Tuberkulöser mit Gesunden. So führt Robert Koch als wichtigsten Punkt die mangelhaft gelüfteten und kleinen Schlafräume für die Utbertragung der Tuberkulose an. Von den in ihren Wohnungen verstorbenen Phthisikern hatten damals 40\% eine Einzimmerwohnung und 41,7\% Zweizimmerwohnungen. Ja, Seiler stellte fest, daß fast $30 \%$ sïmtlicher 1914 besuchten Lungenkranken genötigt waren, ihr Lager mit anderen Personen zu teilen. Müller konnte auf Veranlassung von Hirsch finden, daß $60,7 \%$ der in Göttingen an Tuberkulose Gestorbenen in noch nicht 5\% aller Häuser gewohnt haben. Dasselbe erhellt aus den Feststellungen Sievekings über den Stand der Tuberkulose in Hamburg, wo vor dem Kriege in 
dem wohlhabenden Bezirk der Uhlenhorst 6-8 Tuberkulose-Erkrankungen gegeniiber 17-19,9 Tuberkulose-Erkrankungen pro 10000 in den enger bewohnten Stadtteilen kamen. Ebenso spiegeln sich in einer anderen Arbeit Sievekings die günstigen Folgen solcher die öffentliche Hygiene fördernder Vorgänge in einer schnelleren Abnahme der Tuberkulose in Hamburg nach Aufhebung des Befestigungsgürtels, nach Einäscherung der durchseuchten Stadtteile durch die große Feuersbrunst 1842 und nach Anlage der Kanalisation. Es steht eben der Tatsache, daß der größte Teil aller Menschen in Kulturgebieten einen tuberkulösen Infekt erleidet und ihn glatt überwindet ohne zu erkranken, die andere Tatsache gegenüber, daß selbst der kräftigste Organismus einer massigen Infektion erliegen kann. Preuß sagt: „Die Intensität und Ausdehnung der Infektion ist der Verdünnung resp. der Menge des inhalierten Virus proportional." Ebenso kommen nach Lubarsch für die Wirkkamseit der Bakterien in Betracht: Zahl der eindringenden Bakterien, ihre Virulenz und Herkunft und Häufigkeit der Infektion. Hiermit steht die Tatsache im Einklang, daß die Schwindsuchtsabnahme, wie Koch feststellte, schon eine Reihe von Jahren angedauert hat, ehe die neuen Behandlungsmethoden einen beachtenswerten Umfang angenommen hatten und auch in anderen Ländern des Kontinents sowie der neuen Welt ohne die gleiche soziale Fürsorge wie in Deutschland eintrat und auch Ranke meint, daßB die Abnahme der Tuberkulose vor dem Weltkrieg weniger die Folge bewährter, gegen diese Krankheit gerichteter Bekämpfungsmaßregeln als eine Begleiterscheinung des wirtschaftlichen Aufschwungs sei. Ebenso machte Reiche für den erheblichen Morbiditätsüberschuß der Belasteten an Hand eines großen Materials die gehäuften Ansteckungen verantwortlich. Uther diese Einflüsse liegen Erfahrungen in solchem Umfange und bereits seit so langer Zeit vor daß sie von der Wissenschaft und Praxis voll ausgewertet werden konnten, und die Perspektive, die uns durch die neuerdings entstandene, katastrophale Wohnungsnot geboten wird, nur leider allzu scharf beleuchtet ist. Anders liegen die Verhältnisse, die durch die Art der Kriegsführung und die Ausdehnung wirtschaftlicher Mißstände in erster Linie für die am schwersten betroffenen deutschen Länder und weiter auch in den durch die wirtschaftlichen Schwierigkeiten in merklicher Weise in Mitleidenschaft gezogenen Nachbarstaaten geschaffen wurden. Eine Schwächung der Widerstandsfähigkeit breitester Massen durch die grausamen Entbehrungen, die uns die Blockade gebracht hat, lag eigentlich nicht mehr im Bereich der Möglichkeiten unserer Kulturstufe und sozialen Fürsorge; und so waren wir über ihre Folgen, die wir wohl vermuten konnten, nicht so im klaren, wie wir es bezïglich der vorher genannten Schädlichkeiten sind. Wir kommen damit zu dem für unsere vorliezenden Resultate sicher am meisten im Vordergrund stehenden Schädigungsmoment, das ist die Schwächung der Resistenzfähigkeit des einzelnen. Leider haben uns die letzten Jahre hierfür ein überreiches Material geliefert. Wie bei allen Tuberkulosefragen, ist es im Rahmen eines kleineren Aufsatzes nicht im entferntesten möglich, alle wertvollen diesbezüglichen Angaben zu berühren, nur so viel sei festgestellt, daß sie alle die Tatsache belegen, daß im entkräfteten Organismus dieTuberkulose aufflammt und um sich greift wie ein Herdfeuer, das die schützende Wand des Herdes sinken sieht. Im Felde zeigten gewöhnlich nur solche Soldaten 
den Ausbruch einer latenten Tuberkulose, die außergewöhnlichen Anstrengungen, mangelhafter Ernährung und erschöpfenden Krankheiten ausgesetzt waren und andererseits haben die großen Entbehrungen in der Heimat eine ständige Zunahme der Tuberkulose bedingt (Franz, Leschke). Lubarsch hält es für wahrscheinlich, daß hierbei die durch die Unterernährung bedingte, starke Zerstörung der roten Blutkörperchen von größter Bedeutung ist; eine Vermutung, die auch von anderen Autoren geäußert und sehr überzeugend ist und für das Verständnis tuberkulöser Erkrankungen einen wertvollen Fingerzeig gibt. Ebenso wie die Hyperämie der Entwicklung tuberkulöser Prozesse einen erheblichen Widerstand entgegensetzt, disponiert die Anämie zur Tuberkulose. Wenn wir dann in der primären Anämie das äußere Zeichen eines Mißverhältnisses zwischen Leistung und Kraftvorrat oder zwischen notwendigem Bedarf und Belieferung mit Kraftmitteln erblicken, so finden wir ein einfaches, klares Kausalverhältnis zwischen der Utberlastung weitester Volksschichten bei gleichzeitiger Unterernährung und ihrem Einfluß auf die Ausbreitung der Tuberkulose. Gleichzeitig haben wir in der Beschaffenheit der Blutzusammensetzung einen sinnfälligen Titer der Widerstandskraft des Organismus. Und zwar stellt es Lubarsch als wahrscheinlich hin, daß die chronische Unterernährung begünstigende Bedingungen, sowohl für das Haften wie für das Fortschreiten der tuberkulösen Infektion schafft. Die Umkehrung der.Gleichung zeigen uns die Feststellungen aus der Zeit vor dem Kriege, die, wie u. a. Ritter einen Rückgang der Mortalität fanden, den Ritter jedoch nicht dem Rückgang der Morbidität gleichsetzt; sondern es hatte im wesentlichen die Lebensdauer zugenommen. Und zwar betraf diese Zunahme die leichten Fälle durch eine Abnahme der Aktivität. Jetzt wiederum kommen die Autoren fast ausnahmslos zu demselben Ergebnis, daß die durch den Krieg bedingte Zunahme der Tuberkulose $\mathrm{zu}$ einem großen Teil in durch Verminderung der Widerstandskraft manifest gewordenen, alten Tuberkulosen besteht (Leschke). Bliedung fand im Jahre 1918 den Prozentsatz der Fälle, die längere Zeit hindurch latent waren und erneut Aktivierungssymptome aufwiesen, um weit über die Hälfte größer als im Jabre 1913/14. v. Hayek illustriert den Modus hierbei folgendermaßen: „Die latente Tuberkulose ist in Wirklichkeit keine ruhende, inaktive Tuberkulose, sondern eine Tuberkulose, die unter stetem Kampf von der Durchseuchungsresistenz in Schach gehalten wird. Wie wahr diese in der für das Verständnis der Tuberkulose überragend wertvollen Arbeit v. Hayeks immer wieder in den Vordergrund gestellte Tatsache ist, können wir daraus ersehen, daß Lubarsch bei 14,3\% der Fälle mit geheilter Tuberkulose und Verkalkungsherden lebende Tuberkelbazillen im Blut nachweisen konnte. v. Hayek gibt dann weiter an, daß diese Herde mit Heilungstendenz gleichzeitig Zentren immun-biologischer Abwehr darstellen, also für den Organismus unter normalen - sit venia verbo - Bedingungen sogar von Vorteil sind, ebenso wie etwa für ein Heer von genügender Stärke ein Feind, der zur Niederhaltung ständig kleiner kriegerischer Unternehmungen bedarf, nur eine vorteilhafte Ubung bedeutet und den Abwehrapparat vor dem Einschlafen und sch wächender Tatenlosigkeit bewahrt. Diese gute Seite der Lage hat jedoch nur solange Geltung, als die statische Immunität (Müller) eine entsprechende Höhe hat. Ândert sich das Kräfteverhältnis (statische Immunität) durch Herab- 
setzen der Durchseuchungsresistenz des Organismus (negative dynamische Immunität, Müller) bei Unterernährung und Erschöpfung, dann verliert die bis dahin gefesselte Tuberkulose keine Zeit, sondern auf den ersten Schritt, den die Abwehrkraft zurückweicht, tritt die ständig mobile Truppe ihren Vormarsch an. Hiermit ist aber auch wieder andererseits die Erfahrung in Einklang zu bringen, daß viele Mannschaften, die sich mit ausgeheilten, inaktiven Prozessen gestellt haben, den Felddienst gut vertrugen, oft sogar unter Kräftigung ihrer Konstitution (Ru mpel, zitiert bei Tachau). Diese Beobachtungen stammen aus dem Jahre 1916 und betreffen offenbar die Kranken, welche nur solchen zu ihrer Leistungsfähigkeit im Verhältnis stehenden Anforderungen genügen mußten und im übrigen gute Verpflegung gehabt hatten. v. Hayek empfiehlt für gewisse Formen der Tuberkulose körperliche Ausarbeitung, die jedoch nur dazu dienen darf, die Tätigkeit der tuberkulösen Prozesse soweit anzuregen, daß sie ihrerseits den gut belieferten Abwehrapparat üben und stählen, ohne ihm gefährlich $\mathrm{zu}$ werden. Dieses optimale Verhältnis ist nun, wie gesagt, durch Nichterfüllung der wichtigsten Vorbedingung, das ist genügende Errnährung und Vermeidung übertriebener Anforderungen, schwer gestört. Wieweit die Unterernährung im Volke gegangen ist, erhellt aus den genauen Angaben Fahrs über die Durchschnittsgewichte vor und nach dem Kriege, wobei eine grundsätzliche Gewichtsabnahme des einzelnen um eine. Anzahl Kilogramm herauskommt, die bei einzelnen Fällen soweit geht, daß sie zur direkten Verhungerung geführt hat. Den Einfluß dieser Verhältnisse auf die Tuberkulose fand Gruber ebenfalls weniger in einer Verschiebung der Kurve der Tuberkulose im ganzen, als darin, daß die Reihe der leicht verlaufenden Fälle noch einen bedeutend bösartigeren Verlauf nehmen als früher, so daß die Zahl der Todesfälle an Tuberkulose im Kriege wesentlich gestiegen ist. Hierbei weist nach ihm ein deutlicher Unterschied zwischen Stadt und Land zuungunsten der Stadtbevölkerung auf den Einfluß der Ernährung in erster Linie. Hamel fand, daß die Tuberkulose-Mortalität auf dem Lande um $40 \%$ in der Stadt um 60\% zugenommen hat. Die interessantesten Feststellungen, die ich über den Einfluß der Unterernährung auf die Tuberkulose-Verbreitung finden konnte und die in jeder Hinsicht einen Parallelismus zu den heutigen Verhältnissen zeigen, bringt Lindées in seiner. Arbeit: Untersuchungen über den Einfluß von Notjahren auf die Tuberkulose-Häufigkeit, sowie die Latenz der Tuberkulose auf Grundlage der Kassationen beim finnischen Militär. Er schildert die Verhältnisse etwa folgendermaßen: Unter mehreren Jahren mit Mißernten trat die schwerste Hungersnot 1866/68 ein. Die Zahl der Geburten sank. 20 Jahre später stieg ohne anderen Grund die Kassationsziffer (Zurückstellungsziffer) für Tuberkulose von 6,3\% auf $14,7 \%$, während die Anzahl der Besichtigten abnahm. Dabei verläuft die Steigerung der Kassationen parallel der Tuberkulose-Frequenz und die größte Steigerung im Jahre 1888 steht mit dem schwersten Notjahr 1867 in Verbindung. Die Erklärung, die Lindées 1909 für diese Tatsache gibt, ist so auffallend passend für unsere Zeit, daß ich sie in Kürze hier wiedergeben möchte: „Da es sich nicht leugnen läßt, daß ein schwacher, schlecht genährter Organismus leichter der Tuberkulose zum Opfer fällt als ein kräftiger, gut genährter, so müssen in Zeiten, in denen Mangel an den notwendigsten Nalirungsmitteln herrscht und man 
genötigt ist, diese mit Surrogaten von geringem Nährwert zu ersetzen, in noch höherem Grade kraftlosere, gegenïber der Tuberkulose widerstandsschwächere Organismen erzeugt werden. . . . Denn durch diese den Organismus schwächenden. Umstände wird sowohl die Lokalisation der Tuberkulose-Infektion und ihr Úbergang in manifeste Formen erleichtert. . . . Es wird sich zeigen, daß diese Gesetze auch gerade für unsere Zeit volle Geltung haben. Selbst nach dem kurzen Kriege 1870/71 ohne die einschneidenden wirtschaftlichen Folgen für die Heimat konstatiert Ranke, daß die Tuberkulosezahl im Jahre 1872 rund um $1 / 10$ höher lag als vordem. Den wichtigen Einfluß von Erschöpfungszuständen, die bei uns ebenfalls auch nicht unerheblich mit ins Gewicht fallen, erläutert Pieper, der bei ejner sehr großen Zahl tuberkulöser russischer Kriegsgefangener feststellen knnnte, da 3 ïber $1 / 4$ meist vor dem Kriege oder während desselben schwere, schwächende Krankheiten durchgemacht hatten.

Wenı wir sodanı vor dem Eintreten in die Behandlung der Tuberkuloseverteilung und Zunahme in den einzelnen Altersabschnitten an Hand unseres Materials noch einen kurzen Blick auf die Tuberkulosekurve ïberhaupt aus der Zeit vor dem Kriege werfen, so sehen wir, daß die Tuberknlose-Sterblichkeit etwa um die Mitte des vorigen Jahrhunderts, und zwar nicht nur in Deutschland einen gewissen Höhepunkt erreicht hat (Robert Koch, Werner). Von da ab sinkt sie langsam und stetig wieder. Robert Koch gibt an, daß in Preußen bis 1866 die Tuberkulose ein wenig oberhalb 30,3 pro 10000 war, und dann die Zahl im Jahre 1908 bis auf 16,24 pro 10000 , also nahezu um $50 \%$ gesunken ist. Diese Abnahme läßt sich fast in allen Kulturstaaten, auch der Nenen Welt, welche diesbezügliche Aufstellungen besitzen, verfolgen; bis auf wenige Staaten wie Irland, Norwegen und Japan, die auch dinn noch eine aufsteigende Kurve zeigten. Wir müssen also für die Propagation der Tuberkulose noch eine Reihe anderer Faktoren in Rechnung setzen als die bisher erörterten, und zwar ist hier wohl daran zu denken, daß diese Lünder wahrscheinlich lange bis zu einem gewissen Grade tuberkulosefrei gewesen sein werden und ums dann vergegenwärtigen, daß in tuberkulosefreien Ländern die Tuberkulose als akute Seuche auftritt (Krause). Dieses dürfte mutatis mutandis auf die genannten Länder zutreffen. Im übrigen betrug nach Seiler der Riickgang der Tuberkulose in Preußen an Orten von 15000 Einwohnern und mehr von 1880 bis $191253 \%$, während der Rückgang der allgemeinen Sterblichkeit $45 \%$ betrug, und zwar sank die Tuberkulose-Sterbeziffer noch in den Jahren von 1907 bis $1912 \mathrm{um} \mathrm{16,2 \%}$, die allgemeine um 14,4\%. Der Abfall der Tuberkulosekurve geht in fast allen deutschen Statistiken bis zum Jahre 1913, so auch in Bayern (Gehrhardt). In Österreich scheint der Anstieg etwas länger angedauert zu haben und hat dort ja auch mit 35 pro 10000 in dieser Zeit ungefähr die größte Höhe in Europa erreicht; aber auch dort findet sich von 1906 bis 1913 ein Abfall, dann wieder zunächst ein langsames, seit 1916 rapides Ansteigen der Tuberkulose-Todeszahlen (Pick). Wenn wir auch anfangs der Tatsache gerecht wurden, daß die Tuberkulose bereits zu einer Zeit zurückzugehen begann, als ihre Bekämpfung auf wissenschaftlicher Basis noch nicht in größerem Maßstabe eingesetzt hatte, so werden wir für dic auffallend schnellere Abnahme in den Jahren 1907/1913 trotz der rapide zunehmenden Bevölke- 
rungsdichtigkeit mit größter Wahrscheinlichkeit die Wirkung ärztlicher und sozialer Fürsorge erblicken können. Die einzige mir bekannte Mitteilung, daß die Tuberkulose-Mortalität unabhängig von der Wohlfahrt der Bevölkerung sei, stammt von de Jäger aus dem Jahre 1905 für die Provinz Friesland. Hierbei müssen wir die Tatsache berücksichtigen, daß nach den Auseinandersetzungen Robert Kochs dic Wohlfahrt eines Landes natürlich nur dann mit einer günstigen Beeinflussung der Tuberkulose zu identifizieren ist, wenn sie eine entsprechende fortschreitende Wohnungshygiene bedingt, was bei den konservativen Sitteú weiter bäuerlicher Kreise sicher nicht immer der Fall ist. Es kann z. B. das Beibehalten der kleinen Schlafräume oder womöglich eingemauerter Alkoven die notwendigsten hygienischen Forderungen trotz einer gewissen Wohlhabenheit von vornherein in Frage stellen.

Das erfreuliche Bild des offensichtlichen Rückganges der Tuberkulose wurde dann durch die elementare Umwälzung, die uns der Ausbruch des Weltkrieges brachte, jäh unterbrochen und prompt in das Gegenteil gewandelt. Die Kurve der Tuberkulose-Mortalität beginnt mit dem Jahre 1914 wieder zu steigen, und zwar bemerkenswerterweise nicht nur in Deutschland, sondern auch in Holland und anderen Nachbarländern (Saltet, Saget). Wir haben nach Prinzing, der die Veröffentlichung des Reichsgesundheitsamtes bringt, eine Zunahme der Tuberkulose-Todesfälle um fast $1 / 3$, zum Teil fast die Hälfte, so daß wir nach Samson 1917 bereits den Stand von 1889 wieder erreicht hatten. Nach anderen Angaben stieg die Mortalität der Zivilbevölkerung um 1/3, die der Tuberkulose um das Doppelte. Wenn wir nun diese Verhältnisse etwas genauer analysieren und damit zu dem ersten Abschnitt unserer speziellen Untersuchungen, der Kindertuberkulose übergehen, so bleibt zu dem vorigen ergänzend zu bemerken, daß bereits vor dem Kriege in einem eigentümlichen Gegensatz zu dem stetigen Abfall der Tuberkulosekurve im ganzen die Kurve der kindlichen Tuberkulose stand. Wir finden nämlich, daß diese schon damals nicht nur nicht an der allgemeinen Abnahme teil hatte, sondern eine weitere Zunahme der Sterbeziffer aufwies. Nur ganz wenige Bezirke, wie z. B. Hamburg und München, konnten auch hier eine günstige Entwicklung aufweisen (Sieveking, Bartschmidt). Zwar scheint Thiele im Vergleich der Zeit von 1881 bis 1885 zu der Zeit von 1907 bis 1911 auch eine gewisse Abnahme der Kindertuberkulose gefunden zu haben; doch ergeben die medizinal-statistischen Mitteilungen des kaiserlichen Gesundheitsamtes bei dem Vergleich der Jahre 1898 bis 1902 mit 1903 bis 1907 eine offenbare Zunahme der Kindertuberkulose; ebenso äußern sich Kirchner, Behla und Hamburger. Vielleicht läßt sich diese Tatsache zum Teil dadurch erklären, daß trotz der Hebung der allgemeinen Lebensbedingungen unter Unterstützung ärztlicher Maßnahmen auch in den letzten Jahren vor dem Kriege durch die gleichzeitige gewaltige Zunahme der Bevölkerungsdichtigkeit die-Möglichkeit einer früheren Infektion ganz erheblich gestiegen sind. Denn wenn auch die Tuberkelbazillen sicher nicht ubiquitär sind, so liegt es doch sehr nahe anzunehmen, daß durch die in der jetzigen Form als Errungenschaft der letzten Jahrzehnte anzusehenden Verhältnisse des öffentlichen Verkehrs und den Zulauf zu den großen Städten für jeden Großstädter zum mindesten die Gefahrzone erheblich vergrößert ist. Denn, obschon die Möglichkeit einer germinativen Ubertragung erwiesen 
ist, v. Baumgarten fand sogar eine verhältnismäßig häufige Erkrankung der Plazenta, so spielt diese doch sicher nur eine untergeordnete Rolle. v. Behring sagt: „Es gibt keine individuelle Disposition, sondern nur eine Art-Disposition.“ Diet1, Moltrecht und andere fanden, daß bei Neugeborenen tuberkulöser Eltern keine Tuberkulinreaktion hervorzurufen ist. Dietl vermutet allerdings doch, daß eine gewisse Disposition von den Eltern auf die Kinder übertragen wird, möglicherweise aber gleichzeitig eine gewisse Immunität, da die Kinder gesunder Eltern oft schwerer und ernster erkranken (ähnlich Krause). Im Vordergrunde steht jedoch bei weitem die Infektion durch Inhalation und Fütterung, Gehrels führt die während des Krieges beobachtete Zunahme der Mesenterialdrüsentuberkulose auf schlechte Überwachung der Mijchproduktion und cine häufigere Infektion mit dem Typus Bovinus zurïck. Aber nicht nur die Mesenterialdrüsentuberkulose hat nach dem Kriege im Kindesalter stark zugenommen, sondern auch alle anderen Erkrankungsformen. Ich setze den weiteren Erörterungen den Ausspruch Beh rings voraus: ,Die menschliche Lungenschwindsucht ist bloß das Ende vom Liede, welches dem Schwindsuchtskandidaten schon an der Wiege gesungen wurde", da derselbe, trotzdem man hier mit Recht einige Einschrünkungen gemacht hat, eine große Wahrheit birgt. Auch Dietl sagt, daß die Infektion in der Kindheit einen bestim. menden Einfluß auf die Erkrankung der Erwachsenen hat. Wir haben dieses evident aus den besprochenen Angaben Lindées über die Tuberkulose-Zunahme 20 Jahre nach der Hungersnot in Finnland gesehen. Es muß uns also die Tatsache einer dauernden weiteren Zunahme der Kindertuberkulose, wie wir sie vor und besonders nach dem Kriege beobachten, am allermeisten zu denken geben, und zwar meines Erachtens nicht nur als pessimistische Betrachtungsweise, sondern ganz im Gegenteil, deshalb weil wir hier für unsere Arbeit auf Erfolg rechnen dürfen, selbst unter den derzeitigen denkbar ungünstigen äußeren Bedingungen, die uns die Hände bezüglich der Durchführung der erprobten Grundsätze im Kampf gegen die Tuberkulose einfach binden. Wir müssen uns nur mit dem Gedanken vertraut machen, andere, nene Behandlungsmethoden in weitestem Umfange zur Anwendung zu bringen. Für das Verständnis von dem Wesen der Tuberkulose ist die Kindertuberkulose deshalb von besonderer Bedeutung, weil sie uns in der reinsten Form die Reaktion des Körpers auf den tuberkulösen Infekt zeigt und weil wir an ihr am ehesten den Gang der Infektion ersehen können. Zunächst müssen wir hier berücksichtigen, da $B$ die Tuberkelbazillen möglicherweise eine Zeitlang latent im menschlichen Organismus verweilen können, $d$. h. ohne spezifische histologische Veränderung zu setzen (v. Baumgarten, Bartels, Moltrecht u. a.) ${ }^{\mathbf{1}}$ ). Wir können jedoch wohl so viel daraus ersehen, dal3 die Tuberkelbazillen häufig nicht gleich an der Eintrittsstelle in den Organismus spezifische Veränderungen

1) So meinen Barthel und Moltrecht, daß auch die latente Tuberkulose analog den pyogenen Infektionen ein Stadium erzeugen kann, das lediglich durch allgemeine Reizerscheinungen charakterisiert ist, die er im Sinne eines Immunisierungsvorganges deuten möchte. Ja er fand sogar, daß den histologischen Veränderungen ein sogen. lymphoides Stadium vorangeht, in welchem das gesamte regionäre Lymphdrüscnsystem (des Verdauungs- und Respirationstraktus von der Tuberkelbazilleninvasion betroffen ist Hamburger steht dieser Auffassung skeptisch gegenüber). 
setzen, sondern wie z. B. beim Kinde, zunächst oft nur eine manifeste Erkrankung der regionären Lymphdrüsen erfolgt (Lubarsch, Kretz, Krückmann, Orth, Weigert, Koch und Möllers). Wenn es jedoch zur manifesten Erkrankung gekommen ist. so verläuft diese, wie Ibrahim und Ranke zeigten, in den ersten beiden Lebensjahren vorwiegend deletär, so daß Ibrahim drekt äußert: Die Zahl der Todesfälle im 1. Jahr ist identisch mit der Zahl der Erkrankungen; er lehnt daher den Begriff der latenten Säuglingstuberkulose ab. Diesen scheinbaren Widerspruch können wir nun, glaube ich, dadurch erklären, daß es pathologisch-anatomisch wohl nur eine tödliche säuglingstuberkulose gibt. Die geringe Widerstandskraft und das Fehlen der erworbenen Immunität erklären dieses (Ranke). Bandelier und Röpke sagen, daß im Säuglingsalter das Lymphsystem dem Ansturm der eindringenden Bazillen nicht gewachsen sei. Beim Kleinkinde dagegen vermögen die Drüsen die Bazillen besser mit Beschlag zu belegen und die Infektion vom übrigen Körper zeitweise oder dauernd abzuhalten. Ich möchte hier, wie in allen diesen Fragen, das Schwergewicht auf den gesamten Organismus legen, dessen Durchseuchungsresistenz dann eben imstande ist, den Prozeß zu lokalisieren. Ich glaube nämlich auch aus Gründen, auf die ich später noch zu sprechen komme, daß der Widerstand, den das zarte Lymphsystem des Kindes ohne indurierende Anthrakose usw. dem Fortschreiten des tuberkulösen Prozesses entgegenzusetzen vermag, nicht allzu bedeutend ist, wofür die schnelle Verbreitung auch von den Drüsen direkt auf die Lunge bei mangelhafter allgemeiner Resistenzfähigkeit sprechen dürfte. Während diese besonderen Bedingungen, unter denen der Säugling in den Kampf mit der Tuberkulose tritt, maßgebend für die Form und Lokalisation der tuberkulösen Prozesse dieser Zeit sind, zeigen die interessanten Versuche Backmeisters, daß wiederum die Vorgïnge im Kindesalter ihrerseits dann den Charakter der Tuberkulose in späteren Jăhren bestimmend beeinflussen. Es war ihm nämlich nur unter der Bedingung möglich, beim Tier der menschlichen Lungenschwindsucht ähnliche Tuberkuloseformen zu erzeugen, wenn bereits ältere Tuberkuloseherde vorhanden waren. Wie der Verlauf einer jeden Infektion bedingt wird durch zwei Hauptfaktoren, nämlich a) die Stärke des Angriffs des Krankheitserregers und b) die entgegenstehenden Abwehrkräfte, so sind die letzteren wieder die Resultante aus der allgemeinen Widerstandsfähigkeit des betreffenden Organismus und dem Grade der Entwicklung seines in Betracht kommenden spezifischen Abwehrvermögens. Die geringe Entwicklung des letzteren bedingt in erster Linie die Form der Kindertuberkulose, durch welche der Körper erst in den Besitz dieses spezifischen Abwehrvermögens kommt. Durch die so erworbene Durchseuchungsresistenz wird der Charakter der rezidivierenden Tuberkulose, und die Tuberkulose des Erwachsenen ist ja fast immer als Rezidiv anzusehen, in dem Simne beeinflußt, daß foudroyante Verlaufsformen, wie in der frühesten Kindheit, zu den Seltenheiten gehören. Die Abwehrkraft des Körpers reicht dann aus, den Prozeß im schnellen Fortschreiten zu hemmen, so daß das gewöhnliche Bild der Lungenschwindsucht entsteht. Da diese, wie gesagt, beim Kinde nicht vorliegt, sehen wir im frühen Kindesalter nie ausgeheilte oder vernarbte Herde (Bandelier und Röpke). Zweifellos den wichtigsten Einfluß auf die Verbreitung der Kindertuberkulase scheint wun die jeweilige Infektionsmög- 
lichkeit und Gelegenheit zu haben. Hamburger fand, da B bei genauerem Nachforschen regelmäßig die Berührung mit einem Tuberkulosekranken anamnestisch für die infizierten Kinder aufzudecken war. Auch hier tritt der einschneidende Unterschied zwischen den Volksschichten verschiedener wirtschaftlicher Stellung hervor und zeigt uns zugleich die Quelle für die sich oft erst im späteren Leben manifestierenden zahlreichen Erkrankungen. Während bei ärmeren Kindern bis zum 14. Lebensjahre annähernd $100 \%$ als infiziert gelten können (von Behring, Hamburger und Ghon, Schmorl, Nothmann, Spaeth), was sowohl durch das geringere Verständnis fïr die Infektionsgefahr als durch die weitaus größere absolute Infektionsmöglichkeit bedingt ist, finden wir bei besser situierten Kreisen die Kindertuberkulose als Seltenheit (Schloßmann). Daß auch diese im weiteren Leben nicht verschont werden, geht aus den Untersuchungen Selters an Bonner Studenten hervor, bei denen in $84 \%$ ein positiver Pirquet gefunden wurde. Aber gerade der friihe Zeitpunkt der Infektion ist deshalb so wichtig, weil in ganz offensichtlicher Weise die Gefährlichkeit der Infektion sehr schnell abklingt, so daß diese nach einer Phase unbedingt tödlichen Verlaufs im Säuglingsalter bereits nach dem 6. Lebensjahre nach Hamburger kaum noch regelmäßig zu einer wirklichen Erkrankung führt, wenn sich natürlich auch ein tuberkulöser Prozeß abspielt.

Aus allen diesen Auseinandersetzungen erhellt die überragende Bedeutung der Kindertuberkulose nicht nur für die Gegenwart, sondern besonders fïr die weitere Zukunft. Und wenn wir noch einmal an unsere bereits geäußerte Ansicht erinnern dürfen, daß der scharfe Gegensatz in den Lebensbedingungen der verschiedenen Gesellschaftsklassen durch den Krieg und seine Folgen bis zu einem gewissen Grade verwischt wurde, und wir alle mehr oder weniger gleichmäßig von den die Propagation der Tuberkulose so sehr fördernden Einschränkungen betroffen sind, so erscheinen unsere Ergebnisse doppelt besorgniserregend. Preuß gibt an, daß die Schwächung zur Zeit der Inhalation des Tuberkulosevirus eine stärkere Ausdehnung der Infektion begünstigt, und zwar namentlich in den Bronchialdrüsen und den Lungen. Wie wichtig erstere im Kindesalter für die Lokalisation der Tuberkulose sind, ist bekannt und erwähnt. So berichten unter vielen anderen Davidsohn, Umber und Keßler über die Zunahme der Tuberkulose-Infektionen und Todesfälle bei Kindern infolge Verschlechterung der äußeren Lage. Davidsohn fand in Berlin, daß die Waisenhauskinder durchschnittlich wesentlich früher infiziert sind als vor dem Kriege. Die Zahl der Infizierten beträgt in den ersten 3 Lebensmonaten z. B. fast $1 / 3$ aller Kinder gegenüber weniger als $1 / 12$ vor dem Kriege. Keßler konnte an Hand der Pirquetschen Reaktion eine Ausdehnung tuberkulöser Infektionen im Kindesalter feststellen, die die Verhältnisse Wiens, welche wir als Stadt mit fast der größten Tuberkulose-Durchseuchung vor dem Kriege kennen lernten, in der Kleinstadt Rostock nicht nur erreicht, sondern übertroffen sind. Fahr konnte in der bereits erwähnten Arbeit eine Zunahme der Tuberkulose, in Prozenten anf die Gesamttuberkulose berechnet, fïr die Zeit von 1914-1918 eine steigende Reihe von $9,5 \%, 13,8 \%, 17,5 \%, 18,3 \%$, $19,9 \%$ feststellen. Für das Jahr 1919 und das erste Halbjahr 1920 fand ich noch eine weitere Zunahme auf $20,2 \%$ und $20,3 \%$ für das Alter von 0 bis 
15 Jahren, also eine Zunahme von nunmehr rund 100\%. Der Versuch, unser Material auf die einzelnen Jahresklassen verteilt $\mathrm{zu}$ verarbeiten, mußte wegen der relativ geringen Anzahl der Kindersektionen im Alter von 6-15 Jahren für dieses Alter aufgegeben werden, jedoch zeigen unsere Protokolle gleichlautend mit den klinischen Ergebnissen anderenorts eine sicher gestellte gewaltige Zunahme in den ersten 4 Lebensjahren.

$\begin{array}{lrr} & 1915 & 1919 \\ \text { bis 1 Jahr } & 7,3 \% & 9,4 \% \\ 1-2 \text { Jahre } & 13,4 \% & 28,0 \% \\ 2-3, " & 13,3 \% & 16,0 \% \\ 3-4, " & 14,2 \% & 46,0 \% \\ 4-6,, & 13,0 \% & 21,7 \%\end{array}$

(auf die Gesamtzahl der Sektionen in den betreffenden Jahresklassen berechnet).

Hierbei müssen wir betonen, daßB diese Angaben nur in diesem Zusammenhange ihre Geltung haben, da sie nur einen Ausschnitt aus dem Hamburger Staatsgebiet wiedergeben, das vor dem Kriege besonders günstige Verhältnisse auch bezüglich der Kindertuberkulose bot.

Wir sehen also, wie die Offensive der Tuberkulose folgerichtig an der schwächsten Stelle ihrer gegnerischen Front angesetzt wurde. Dieser Frontabschnitt, der auch im Frieden noch in fließender Bewegung begriffen war, ist nunmehr durchbrochen und mit dem Umsichgreifen der Kindertuberkulose sehen wir die Gefahr der Aufrollung weitester Teile unserer Kampflinie heraufziehen. Da der kindliche Organismus bei dem vollkommenen oder relativen Mangel immun-biologischen Schutzes fast ausschließlich auf die durch die Kraft seiner Konstitution bedingte Komponente der Durchseuchungsresistenz angewiesen ist, ist durch schwerste Schädigung der letzteren infolge Unterernährung die momentane Widerstandsunfähigkeit zu erklären. Wie direkt die Erkrankungskurve hier mit der jeweiligen Ernährungslage zusammenhängt, geht aus den neuesten Veröffentlichungen Bartschmidts hervor. Danach haben wir in München im Gegensatz zu den bisher bekannten Veröffentlichungen, vor allem aus Preußen, mit Ausnahme des Jahres 1916 ein weiteres Sinken der Tuberkulose-Sterblichkeit der Münchener Kind $\%$ verzeichnen, wenn auch der Abfall wesentlich langsamer als vor deri Friege erfolgt. Wir dürften nicht fehlgehen, wenn wir hierin einen Ausdruck del bekannten besseren Verpflegungslage Süddeutschlands, besonders Bayerns, während des Krieges erblicken. Es muß dabei jedoch betont werden, daß in den ersten Lebensjahren, auch in München, eine Zunahme der Pirquetschen Reaktionen zu verzeichnen ist, und die Resultate bei den älteren Kindern durch erhöhte Fürsorgetätigkeit außer der Klinik beeinflußt sind. Im übrigen meint Bartschmidt, daß besonders die Jahrgänge betroffen sind, die als Klein-Kinder mehr oder weniger den Schädigungen der Kriegsjahre ausgesetzt sind.

Wir kommen dann zur Tuberkulose im jugendlichen und reifen Alter. Hier leuchtet es im Verfolg der letzten Utberlegungen ein, daß der jugendliche und reife Organismus, bei welchem die spezifische Immunität durchweg weitgehend entwickelt ist, durch eine Schwächung des Körpers, dem Infekt nicht so hilflos preisgegeben ist als das Kind. Außerdem müssen wir berücksichtigen, daß ür die Erkrankungsziffer im 2. und 3. Jahrzehnt maßgebende Zahl der 
Kindertuberkulore für die jetzige jugendliche Generation tief im Frieden liegt, also ein Finfluß des Krieges sich in dieser Beziehung noch nicht bemerkbar machen konnte. Dem entsprechen auch fast alle darüber erhobenen Befunde in Úbereinstimmung mit unseren Ergebnissen, die eine bemerkenswerte Beeinflussung der Erkrankungen im jugendlichen Alter vorläufig vermissen lassen. Dieses erhellt aus beiliegender Tabelle.

1915

$16-20$ Jahre
$21-30 \quad$,
$31-50 \quad$,

$47,0 \%$

$50,8 \%$
1919

$49,8 \%$

$46,6^{\circ} \%$

$32,2 \%$

(auf die Gesamizahl der Sektionen der betreffenden Altersklassen berechnet).

Wir sind uns darüber klar, daß jedoch auch eine Reihe von Fällen jugendlicher Tuberkulose einen schwereren Verlauf nehmen und haben Beobachtungen hierfür, besonders von klinischer Seite bei früher lungengesunden Soldaten, die während des Heeresdienstes erkrankten. Diese Veröffentlichungen geben gleichzeitig einen wertvollen Beitrag zur Bedeutung der hereditären Belastung für den Verlauf der Tuberkulose. So schreibt z. B. Zadeck:, ,Die primären Erkrankungen und wirklichen Infektionen an Lungentuberkulose im Kriege sind häufig und von ungünstigem Verlauf; die Exazerbationen und Manifestationen der Tuberkulose von Disponierten und konstitutionell Belasteten treten zahlenmäßig und prognostisch mehr in den Hintergrund." Im gleichen Sinne äußerten sich Reiche und v. Hayek. In unserem Material treten diese Fülle jedoch ebenso wie das Soldatenmaterial ïberhaupt vollständig in den Hintergrund und dürften es auch nach den zahlreichen anderen Erhebungen im Vergleich zu der gesamten Volksmasse tun.

Um aber die Hauptverschiebung der Tuberkulose-Erkrankungen weiter zu fixieren, müssen wir gleich einen großen Sprung zur Alterstuberkulose machen. Und wir werden sehen, daß dieser Sprung eine innere Berechtigung hat. Bei genauerem Studium der Verhältnisse finden wir nämlich, daß die Tuberkulosefront auch hier an einer durch den Krieg geschaffenen, schwachen Stelle getroffen ist. Wir hatten im Kindesalter von der noch mangelhaften immunbiologischen Abwehrkraft gesprochen, die dem ganzen Krankheitsbild seinen Stempel aufdrückt. Im Alter haben wir gerade das Gegenteil. Nach dem jahrzehntelangen, soweit siegreichen Kampf des Organismus, da $B$ die Tuberkulose in vollkommener Latenz gehalten wurde bzw. dort, wo sie trotzdem auftrat, unter dem dominierenden Einfluß der Abwehrkraft an schnellem Umsichgreifen behindert worden war, hat die fortschreitende Entkräftung die ohnehin abnehmende, körperliche Kampftüchtigkeit so stark herabgesetzt, daß das Mißverhältnis auch durch die weitgehende Immunität nicht mehr ausgeglichen werden konnte. Wir finden also im Alter zur Zeit eine Form der Tuberkulose relativ häufig vertreten, die wir in diesem Umfange nicht entfernt anzutreffen gewohnt waren. Fahr macht hierauf in seiner erwähnten Arbeit besonders aufmerksam; aus dieser geht gleichzeitig hervor, daß selbst im höheren Alter, wo der Körper sich im allgemeinen entsprechend der geringeren Beanspruchung und Leistungsfähigkeit sein Gewicht auf das Notwendigste reduziert hat, eine durchschnittliche Gewichtsabnahme um 4-5 kg festzustellen ist. 
Ein Rïckblick auf die Tuberkulose-Verbreitung in der Vergangenheit zeigt uns, daß awoh diese auffallende Anderung im Wesen und der Bedeutung der Alterstuberkulose nach ganz bestimmten Gesetzen vor sich geht, die immer wieder von neuem die direkte Abhängigkeit der Ausbreitung und Form der Tuberkulose von der durch die erwähnten zahlreichen Faktoren abhängigen Durchseuchungsresistenz beweisen. Wir sehen nämlich, daß, ebenso wie zu Zeiten des Rüikgangs oder der geringeren Ausdehnung tuberkulöser Erkrankungen (Hebung der allgemeinen Durchseuchungsresistenz), die Alterstuberkulose stark in den Hintergrund tritt, diese bei steigender Erkrankungskurve (mangelhafte Durchseuchungsresistenz) ganz erheblich häufiger wird. Behla fand noch im Jahre 1876 die Tuberkulose über 60 Jahre mit 77,62 pro 10000 der einzelnen Altersklasse vertreten, während sie sich 1912 auf nur mehr 19,81 belief. Und so finden wir vor dem Kriege entsprechend dem günstigen Stand im Kampf gegen die Tuberkulose ein schnelles Absinken der Tuberkulosefälle nach dem 60. Lebensjahre (Reinhart). Dabei handelt es sich zum Teil um alte Herde aus der Kindheit, die wieder aufflackern können, während Fälle frischer Infektion mit Tuberkulose im Greisenalter relativ selten waren (Hoppe Seyler). Auch Schlesinger sagt, daß für einen großen Teil von Alterstuberkulose Infektionen in jüngeren Jahren in Betracht kommen. Selters hält diesen Modus auch im Gegensatz zu Much und Römer für unwahrscheinlich; Daß jedenfalls die Möglichkeit eines Aufflackerns alter Tuberkuloseherde doch recht groß ist, betonten wir bereits eingangs. Und die Annahme Liebermeisters und Rumpfs, daB bei lokalisierter chronischer Tuberkulose die Bakterien in vereinzelten Exemplaren fortwährend im Blute kreisen, wird durch die Untersuchung Lubarschs erhärtet. Alle Befunde der Bakteriologie deuten ja auch darauf hin, daß die Bakteriämie nur dann gefährlich wird, wenn sie entweder durch ihre Massigkeit oder durch mangelhafte Resistenzfähigkeit des Organismus zur Wirkung kommt. So wissen wir zur Zeit, daß bei großen chirurgischen Eingriffen vorübergehende Bakteriämien direkt die Regel sind, während eben nur bei einem deutlichen Mißverhältnis zwischen Angreifer und Abwehrkraft zugunsten des ersteren, ihre deletäre Wirkung diese Tatsache gröber illustriert. Wir können jedenfalls an unserem Material die Wahrscheinlichkeit, daß es sich bei einem großen Teil der Fälle von Alterstuberkulose, wie wir sie jetzt beobachten, um wieder auflebende, alte Prozesse handelt, vollauf bestätigen; wenn wir auch andererseits für die Möglichkeit frischer Infektionen eindeutige Beispiele gefunden haben. Schlesinger wird auch der letzteren Möglichkeit gerecht, indem er darauf hinweist, daß für die in späterem Alter entstehenden oder manifest werdenden Fälle von Tuberculosis pulmonum leichte Infektionsmöglichkeit bei schlechtem Ernährungs- und Allgemeinzustand, schlechten Wohnungsverhältnissen von großer ätiologischer Wichtigkeit sind. Die, Angabe stammt aus dem Jahre 1914 und findet ihre Bestätigung jetzt in so betrübend weitem Maße. Doch blieben derartige frische Infektionen vor dem Kriege große Ausnahmen, so daß die Form der Altersphthise durchweg durch die geringe Ausdehnung der Prozesse mit starker Heilungstendenz ohne Neigung zur Dissemination so ausgesprochen charakterisiert waren, daß sie von allen Beobachtern gleich angegeben werden. Es traten unter dem Einfluß der hoch entwickelten Immunität die Zerfallserscheinungen 
gegenüber reaktiver Bindegewebsentwicklung mit narbig-schiefriger Induration zuriick, so daß nach Schlesinger, Hart, Lubarsch die Aussichten einer „Tuberkulösen Apizitis" im Senium die besten sind. Hart betont andererseits die mechanische Disposition der Spitzen alter Leute durch Verknöcherung der Rippenknorpel, mangelhafte Lüftung und mangelhafte Blut-Lymphzirkulation. Er fand dabei die Lokalisation der sekundären Herde häufig atypisch, aber ohne Neigung zur Dissemination. Selbst frischer entstandene Herde zeigen starke Heilungstendenz. Er erklärt die geringe Neigung zur Dissemination ebenfalls aus der Verödung der Blut- und Lymphbahnen und Verlegung der Bronchiolen. Wenckebach lehnt den entscheidenden Einflul3 der Form der oberen Thoraxapertur und der Verknöcherung der 1. Rippenknorpel auf die Lokalisation der Tuberkulose ab, während die Versuche Backmeisters einen solchen Einfluß deutlich belegen. Das Vorwiegen isolierter tuberkulöser Herde im höheren Alter wird jedenfalls zum Teil dadurch mitbedingt, daß der schnelle Konnex zwischen den Organen besonders auf dem Lymphwege, wie er im jugendlichen Alter auch bei pathologischen Verhältnissen so hervortritt, im Alter durch die genannten Veränderungen bis zu einem gewissen Grade eingeschränkt ist. Charcot, zitiert bei Staehelin, wies darauf hin, daß die Organe im Alter isoliert erkranken und die verschiedenen Läsionen im Organismus keinen Widerhall erwecken. Hoppe-Seyler vermutet in diesen Umständen ebenfalls die Ursache für die Form der Altersphthise: „,Betrachtet man die Nase, den Rachen, besonders die Tonsillen als Eingangspforten für Bazillen, so mag der Schwund dieser Teile, wie er im Alter eintritt, die Verödung der Lymphspalten in dieser Beziehung eine Rolle spielen. Der mögliche Einfluß der Anthrakose auf die Lungentuberkulose bzw. auf den Heilungsverlauf durch Verlegung von Lymphbahnen wird von Lubarsch erwähnt, jedoch läßt dieser die Frage offen, ob die günstige Tuberkuloseziffer in den Kohlengebieten mit der Anthrakose zu erklären ist. Ich glaube auch nicht, daß dieses die einzige Ursache für die an sich überraschende Tatsache ist, daß in den Gegenden mit stärkster Rauchentwicklung die niedrigste Tuberkulose-Sterblichkeit gefunden wird (Gerwiene r), weil gerade für solche regionären Verhältnisse epidemiologische Gründe jedenfalls stark mitwirken. Der Zusammenhang ist jedoch nicht nur in den deutsehen Kohlengebieten, sondern auch in England usw. festgestellt. Nach Baup haben wir in den Londoner Heilstätten die niedrigste TuberkuloseSterblichkeit. Diese Erscheinung läßt sich auch mit den pathologisch-anatomischen Verhältnissen sehr wohl in Einklang bringen. Orth betont das Zurücktreten der tuberkulösen Lymphdrüsenerkrankungen im hohen Alter. Ribbert kommt zu dem Schluß, daß die anthrakotische Induration der Bronchialdrüsen der Mehrzahl nach auf Tuberkulose beruhen. Ich möchte num hieraus nicht den zunächst scheinbar naheliegenden Schluß ziehen, daß anthrakotisch verändertes Gewebe besonders zur Tuberkulose disponiert ist, sondern auf einen günstigen Einfluß der Anthrakose auf die Tuberkulose, wie ihn Lubarsch als gesichert ansieht.

Hellmann fand, daß bei direkter lymphogener Verbreitung der Tuberkelbazillen nach einer Lymphdrüse die tuberkulösen Prozesse in erster Linie in der Peripherie der Drüsen im engsten Anschluß an den Marginalsinus auftreten und bei Einlagerung von Kohlepigment sich andererseits die Kohle- 
partikel in einem frühen Stadium hauptsächlich in den Markpartien der Drüse, oft im nahen Ánschluß an den Drüsenhilus sammeln, also den weiteren Weg verlegen.

Ich möchte num, wie gesagt, in dem Umstand, daß wir in den zahlreichen schwielig-anthrakotischen Herden häufig den Ausgang alter tuberkulöser Prozesse erblicken, eine günstige Wrkung der Anthrakose sehen, denn eine diesbezügliche Beobachtung einer größeren Reihe histologisch untersuchter Hilusdrüsen mit tuberkulösen Veränderungen hat zum mindesten keine Bevorzugung anthrakotischer Drüsenabschnitte von frischen tuberkulösen Prozessen dargetan. Wir fanden im Gegenteil bei 80 Fällen mit tuberkulös erkrankten Lymphdrüsen, die zu allermeist erwachsenen Leichen entnommen waren, die Lymphdrüsen, 3 mal in stärkstem Maße anthrakotisch verändert, 17 mal eine mittlere Anthrakose und $56 \mathrm{mal}$ äußerst geringe anthrakotische Veränderungen, nur bei 4 Kindern noch keine Anthrakose. Dabei zeigt sich deutlich, daß die Tuberkulose in den Drüsen mit starker Anthrakose große Neigung zur Bindegewebsentwicklung in den tuberkulösen Herden zeigt. Die Angabe der Anthrakose bezieht sich jedoch nur auf die mikroskopisch untersuchten Driisenabschnitte, während andere Drüsen andere Verhältnisse bezüglich anthrakotischer Einlagerungen bieten konnten. Maßgebend ist hierfür jedenfalls der Umstand, daß der Kohlenstaub offenbar keine weitere schädigende Reizwirkung setzt im Gegensatz zum Steinstaub, der ja eine verheerende Wirkung als Schrittmacher der Tuberkulose hat (Baup u. a.). Um nach diesem kurzen speziellen Exkurs auf die Alterstuberkulose zurückzukommen, können wir zusammenfassend sagen, daß für die bislang vorwiegend beobachtete lokalisierte oder zirrhotische Form der Tuberkulose a) in der hohen Immunität und b) in der anatomisch bedingten Behinderung einer schnellen Verbreitung der Prozesse hinreichend kausale Momente vorliegen. Wenn wir nun vor dem Kriege eine starke Abnahme der Alterstuberkulose überhaupt und der Verschiebung der größeren Sterblichkeit, die nach Dresen noch in der Zeit von 1881-1885 im 60.-70. Lebensjahre lag, nach dem 25.-30. Lebensjahre für die Jahre 1906-1910 festgestellt haben, so liegen doch, wie bereits gestreift, auch aus dieser Zeit Beobachtungen vor, die die Möglichkeit einer propagierenden Tuberkulose auch in höherem Alter beweisen. Nach Braun und Schlesinger betrug die Sterblichkeit im Greisenalter an Miliartuberkulose 1,5\% gegen 2,5\% der anderen Altersklassen. Ranke sagte 1913, wir sehen im Alter wieder ein wenn auch geringes Ansteigen der generalisicrten Tuberkulose, daneben aber meist sehr chronische Tuberkulose. Er weist für das Zustandekommen generalisierter Tuberkulose auf die Abnahme der Resistenz und Zunahme der Exposition durch den vorwiegenden Zimmeraufenthalt hin. Auch Necker fand bei 600 Sektionen einen relativ hohen Prozentsatz letaler und fortschreitender Tuberkulose, für dessen Verallgemeinerung das Material, das überdies aus Wien stammt, nicht ausreichend sein dürfte. Diese auch im Frieden gelegentlich beobachtete fortschreitende bzw. frische Tuberkulose im höheren Alter ist nun neben der zunehmenden Kindertuberkulose nach unseren Befunden zur Zeit das Charakteristischste in dem Wandel der Tuberkulose-Erkrankungen. Interessanterweise finden wir dieselben Verhältnisse für sich bei der chirurgischen Tuberkulose. D rügg fand auch die chirurgische Tuberkulose, die nach seinen Aufstellungen nach 
dem 50. Lebensjahre früher so gut wie gar nicht zu verzeichnen war, im Jahre 1918 mit $11,7 \%$ vertreten. Ich glaube wohl, daß dieseZunahme der chirurgischen Tuberkulose auch wieder für eine Zunahme der Tuberkulose aufdem Boden alter, abgeheilter Prozesse durch sinkende Widerstandskraft des Organismus zu erklären ist. - Wie sich die derzeitige fortschreitende Alterstuberkulose an unserem Sektionsmaterial dokumentiert, ist am besten aus den vorliegenden Protokollen ersichtlich ${ }^{\mathbf{1}}$.

Aus Raummangel sind die Protokollauszüge gestrichen. Es handelt sich dabei um 33 charakteristische Fälle von frischen Reininfektionen bzw. Neuinfektionen mit Tuberkulose im Alter ïber 60 Jahren.

Bei der Durchsicht derselben fällt zunächst auf, wie wenig diese tuberkulösen Veränderungen klinisch erkennbar waren. Staehelin machte dieselbe Beobachtung im Jahre 1910; die damals allerdings sehr seltenen Fälle waren auch unter anderen Diagnosen ad exitum gekommen, weil sie wegen der uncharakteristischen Symptome, Mangel an Fieber und Schwierigkeit der Perkussion nicht erkannt worden waren. Im übrigen sind dieselben ein deutlicher Beleg dafür, daß hier einerseits in vielen Fällen latente tuberkulöse Prozesse durch die starke Resistenzverminderung von neuem aktiv geworden sind und andererseits aus demselben Grund auch eine Anzahl von neuen Infektionen ermöglicht sein muß, denen durchaus keine besondere Neigung zur Ausheilung eigen ist, wie wir es von früher kennen gelernt haben. von Hansemann wies bereits 1904 darauf hin, daß z. B. die primäre Darmtuberkulose bei verminderter Widerstandskraft oder bei besonderer Disposition auftritt. Der größere Teil der Fälle, von denen wir nur die charakteristischen aufgezïhlt haben, zeigt allerdings ein Wiederaufleben von abgeheilten Spitzenherden mit frischer Aussaat über die bis dahin gesunden Lungenabschnitte, zum Teil mit ganz frischer ausgedehnter Erkrankung der Bronchial- und Hilusdriisen. Daneben stehen einzelne Fälle; die eine neue Infektion ohne das Vorhandensein nachweisbarer alter Herde beweisen. Dieser Beweis ist jedoch überhaupt nur ausnahmsweise zu führen, da in dem hohen Alter Residuen einer früheren Infektion ganz selten vermißt werden. Ich glaube außerdem, die Fälle, die mit einer starken, frischen Beteiligung der regionären Lymphdrüsen einhergehen, mit in den Bereich der Neuinfektionen setzen zu kömnen. Besonders interessant erscheinen einige Fälle, die durch die nachfolgend weiter erläuterten. Tonsillenuntersuchungen eine primäre Tonsillartuberkulose ergaben; diese habe ich jedoch bei der prozentualen Berechnung nicht mit herangezogen, da im Jahre 1915 keine derartigen Untersuchungen vorgenommen waren. Es zeigt ein Vergleich der Fälle mit aktiver Tuberkulose im Alter ïber 60 .Jahren eine Zunahme von $8,3 \%$ des Tuberkulosematerials im Jahre 1915, bis zu 15,6\% in Jahre 1919 und 15,2\% im ersten Halbjahr 1920; also wiederum eine Steigerung um nahezu $50 \%$ der Friedenszahl. Wir dürfen wiederholen, daß dieser Umschwung auf Kosten der stark geschwïchten Widerstands-

1) Auf Wunsch stehen nähere Angaben resp. Protokollauszïge gerne zur Finsicht zur Verfïgung. 
kraft zu setzen ist. Hierdurch haben die noch in .Jahre 1911 von Hart dahin definierten Verhältnisse, daß bei der tuberkulösen Lungenphthise im alternden und gealterten, menschlichen Organismus sich die Verhältnisse der inneren und äußeren Krankheitsursache, auf deren Bedeutung Hueppe hinwies, sehr zugunsten der ersteren verschoben, also eine grundsätzliche Anderung erfahren, und zwar dahin, daß selbst das Lebensalter, welches uns bislang den gutartigsten Verlauf der Tuberkulose zeigte, in steigendem Maße proliferierende und frische Tuberkulosefälle aufweist. Diese Erscheinung im Verein mit der unheimlichen 'Junahme der Kindertuberkulose lehrt uns, daß wir uns also einer neuen Tuberkulosewelle gegenïber befinden, die nach ihrer bisher erkannten Wucht und Höhe wohl geeignet ist, ein gewaltiges Stück unserer Volkskraft hinwegzuraffen.

Nach diesem Überblick ïber die Zunahme und Verschiebung der Tuberkulose-Erkrankungen, durch welche die Ergebnisse Fahrs ergänzt und für die neueste Zeit bestätigt werden, wollen wir noch die Resultate unserer Untersuchungen über den derzeitigen Stand der Beteiligung der Tonsillen an den tuberkulösen Veränderungen anfügen. Diese sind dazu angetan, ebenfalls dic allgemeine Resistenzverminderung des Organismus und dadurch ermöglichte Ausdehnung tuberkulöser Prozesse auch auf sonst nicht so weitgehend beteiligte Organe in größerem Maße nachzuweisen. Die Besprechung der Tonsillartuberkulose führt uns zunächst in das viel umstrittene Gebiet der Frage nach der Eintrittsstelle der Tuberkelbazillen in den Körper und dem Infektionsmodus, den wir bezüglich des Nasen-Rachenraumes genauer besprechen wollen. Wir hatten festgestellt, daß die Tuberkelbazillen ohne Läsion oder Affekt an der Eintrittsstelle eintreten können und, wie z. B. im Kindesalter durchgehend, von den Lymphdrïsen aufgesogen werden (Lubarsch, neuerdings Koch und Moellers), woraus Lubarsch schließt, daß die Lymphdrüsentuberkulose in der Regel auf die Eingangspforte im Wurzelgebiet der Drïsen hinweist. Die Latenz der Tuberkelbazillen ohne histologische Veränderungen ist bewiesen, aber jedenfalls für längere Intervalle nicht maßgebend. Nach Lubarsch muß es als gesichert gelten, daß jede Art fortschreitender Tuberkulose sowohl durch primäre Ansteckung unmittelbar, wie durch eine exogene oder endogene Neuansteckung mittelbar durch. Verbreitung der Tuberkelbazillen auf dem Blut- oder Lymphwege erfolgen kann. Gerade der Umstand aber, daß die Tuberkelbazillen häufig nicht gleich an der Eintrittsstelle nachweisbare Veränderungen setzen, nimmt uns die Möglichkeit, für den stattgehabten Eintritt an dieser oder jener in Betracht kommenden Stelle den direkten Beweis zu liefern, wie wir auch bei den Tonsillen von neuem sehen werden. Schönemann bezeichnet die Tonsillen als subepithelial gelegene, vorgeschobene Halslymphdrüsen, deren Quellgebiet in der Nasenschleimhaut liegt (nach Operationen am Septum häufig lakunäre Angina, Barth). Gegen die Bezeichnung der Tonsillen als Lymphdrüsen wendet sich Loch, der ihre besondere Stellung gewahrt wissen will und darauf hinweist, daß dieselben Endarteriengebiete sind. Diese subepitheliale Lage im Verein mit der von Stöhr beschriebenen Tatsache, daß 3 äberall, wo in Schleimhäuten dichtes adenoides Gewebe unter dem Epithel liegt, normalerweise eine starke Durchwanderung von Lymphozyten durch das Epithel auf die freie Oberfläche stattfindet, weist den Tonsillen 
einen gesonderten Platz in der Reihe der Lymphdrüsen an. Sitöh erkannte denn auch als erster die im Tonsillarepithel vorhandenen Epithellicken als physiologische, präformierte Öfumngen. durch welche dic Lymphozyten hindurchtreten. Verhältnisse, die wir bei günstiger sichnittrichtung sehr schön beobachten kömnen (Abb. 1). Wir sehen dabei vielfach eine fas siebartige Durchlöcherung des Epithels. das in der Tiefo einem dicht kanalisierten Hohwamm

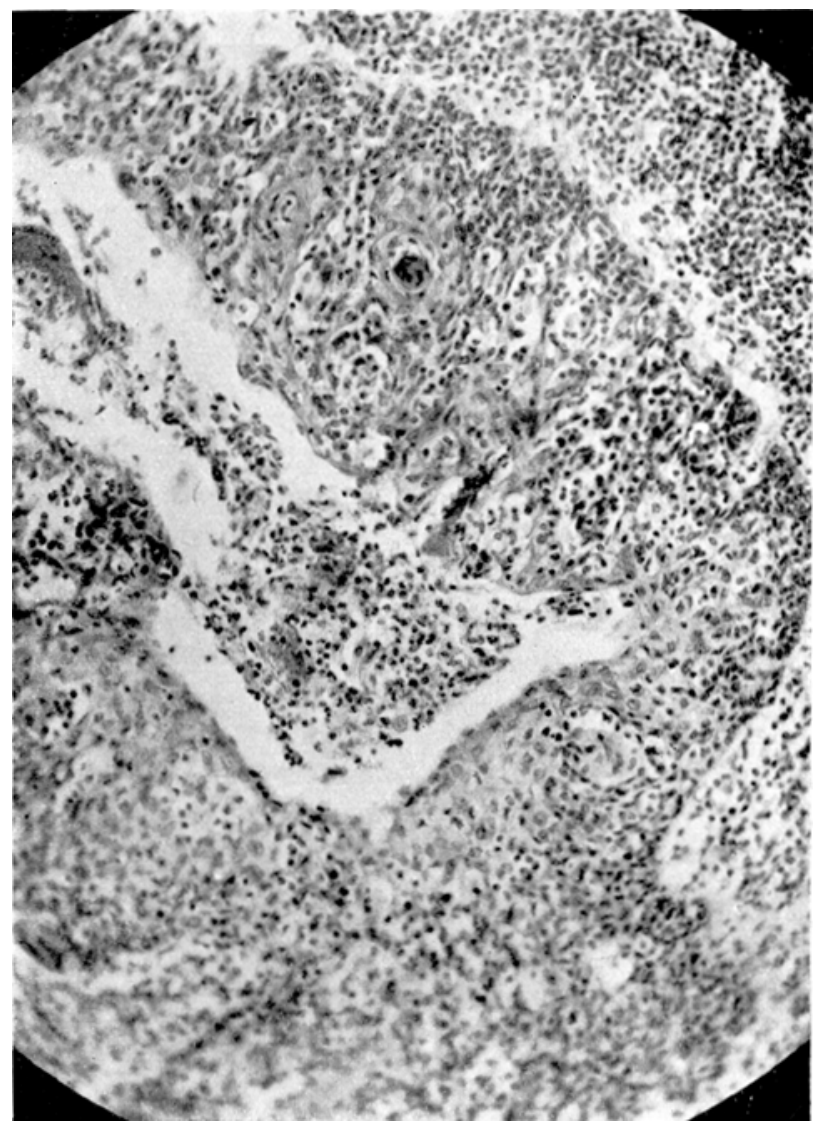

Abb. 1 .

gleichen kamm. Bei der durchaus nicht immer gleichmäBig alle Offummen durchströmenden Lymphoztenemigration entstehen damn. wie siöhr hervorhebt, und auch aus unserer Abbildung ersichtlich ist. häufig Pausen, die fïr das Eindringen von Bakterien aus der Mundhöhle freie Bahn schatfen. Die erwähnte, nach dem Rachen zu gerichtete Strömung verhindert jedoch nach der Ansicht Henkes einen lïngeren Aufenthalt fremder silemente in den Tonsillen. Nun finden wir aher gerade an den 'Tonsillen so ïberaus häufig. ja fast. regelmäßig Veränderungen. die den glatten Ablauf der physiologischen Vorgänge mehr weniger stark beeinflussen. Die Tonsillen werden. Wie bekannt, 
fast regelmäßig bei entzündlichen Erkrankungen des Nasen-Rachenraums stark mitbeteiligt und bereits durch eine leicht entzündliche Schwellung, sowie die damit fast regelmäßig verbundene, reichliche Epithelabschilferung kommt es zur Retention der Ausscheidung der Lakunen. Dieser organisch infektiöse Fremdkörper wirkt in dem nun entstehenden Circulus vitiosus wieder entzündungserregend und fördert die Hypertrophie (Sokolowsky und D mochowsky). Die Abwehrfunktion ist nur solange möglich, als eine ungestörte Zellemigration nach außen erfolgen kann (Barth); und damit sind für die Retention infektiösen Materials die besten Bedingungen geschaffen. König nennt die Tonsillen direkt ein Reservoir entzündlicher Noxen. Dieser Infektionsmodus wurde für die Tuberkulose durch von Baumgarten an Hand von Fütterungstuberkulose bei Kaninchen festgestellt. Und zwar fand er histologisch fast regelmäBig tuberkulöse Veränderungen an den Tonsillen. Aber hierzu braucht es gar nicht zu kommen, sondern wir müssen hier häufig mit der Möglichkeit rechnen, daß die Tuberkelbazillen in den Tonsillen latent verweilen und sie durchwandern, ohne daß dieselben tuberkulös erkranken. Den Mechanismus der Infektion durch den Inhalt des Rachenraumes beschreibt D mochowsky. Beim Schlucken zieht sich die Muskulatur des Pharynx zusammen und drückt, da einige Muskelfasern des Constrictio pharyngis unmittelbar an der äußeren Hautdecke der Tonsillen befestigt sind, ,Sekret" aus den Krypten heraus. Nach dem Schlucken kehrt die Mandel unter dem Einfluß ihres elastischen Bindegewebes in ihre frühere Lage zurück, wobei sich die Krypten weiten und die an ihrer Oberfläche befindliche Flüssigkeit einsaugen. Ein Weg, auf welchem zweifellos die häufigste, nämlich die Sputuminfektion ulzerierender Lungentuberkulose erfolgt. Im gleichen Sinne zeigte Krückmann, daß bei Fütterungstuberkulose nur diejenigen Apparate zu erkranken brauchen, die begierig korpuskuläre Elemente aufsaugen, wie Mandeln und Mesenterialdrüsen, während die Schleimhäute, die in gleicher Weise der Infektion ausgesetzt waren, unverändert bleiben (ähnlich Goedele und Kaiser, zitiert bei Friedemann, sowie Koch und Moellers). Für die primäre Infektion der Tonsillen andererseits, die Orth zuerst bei Kindern beobachtete, müssen wir jedoch auch an die Möglichkeit einer Infektion auf dem Lymphwege aus der Nase, Zahnfleisch usw. denken (Henke). Dieser verfolgte den Vorgang im Experiment an Tieren und Menschen, und zwar beschränkte sich die Lokalisation der von ihm benutzten Rußpartikel ausschließlich auf die Lymphbahnen, wobei die Lymphscheiden der Gefäße allerdings auch stark vertreten waren. Er konnte dann das Weiterwandern der schwarzen Körnchen in kontinuierlichem Strom durch dic ganze Substanż der Tonsille, durch das Epithel nach der freien Oberfläche beobachten. Diese Versuche sind zwar angefochten, aber auch von anderen Autoren bestätigt. Eine zu den Halslymphdrüsen und eventuell zu den Tonsillen aufsteigende Infektion durch perverse Stromrichtung in den thorakalen Lymphwegen, wie sie durch die Arbeiten Zieglers und Tendeloos als prinzipiell möglich dargelegt ist, wird im allgemeinen für sehr unwahrscheinlich gehalten (Bandelier und Röpke). Tendeloo gibt die Möglichkeit an, daß den am meisten kaudalen, tiefen Halsdrüsen während Freßbewegungen Bazillen aus den intrathorakalen Lymphgefäßen zugeführt werden. Ist nun auf dem einen oder anderen Wege das Tuberkulosevirus bis in die Gaumentonsillen und mit diesen 
zu dem lymphatischen Apparat des Nasen-Rachenraumes gelangt, der besonders bezüglich der Rachenmandeln die gleiche oder ganz ähnliche Bedeutung für die Tuberkulose-Infektion besitzt, so daß die Tonsillen auch in erster Linie in diesem Rahmen betrachtet werden müssen, vorgedrungen, so geht sie dinn weiter auf die regionären submentalen und Halslymphdrüsen über (Dmochowsky, Hanau, Krückmann, Koch und Moellers, von Behring, Most, Bandelier, Beckmann, Pertik, Schlenker). Mann macht sogar neuerdings die Tonsillartuberkulose für einen Teil der Fälle von Skrofulose, auch des Gesichts verantwortlich und will gute Erfolge hierbei mit der Tonsillektomie erreicht haben. Eine von hier ausgehende Infektion der Lunge lehnt Most mangels direkter Lymphbahnen ab und auch Beitzke hebt hervor, daß keine zuführenden Lymphgefäße von der Kette der zervikalen Lymphdrüsen zu den bronchialen Lymphdrüsen existieren. Er gibt für die Tuberkulose-Infektion der Lungen von den Halsdrüsen aus den ausschliel3lichen Weg durch die Trunci lymphatici und die obere Hohlvene an und meint, daß dieser Weg wenigstens beim Kinde praktisch ohne wesentliche Bedeutung sei. Die Infektion der Lungen bzw. der Bronchialdrüsen kommt beim Kinde vielmehr in der Regel durch Aspiration von Tuberkelbazillen in den Bronchialbaum zustande; eine absteigende Zervikaldrüsentuberkulose geht manchmal unabhängig davon nebenher. Andererseits konnte Grober Tusche, die er in die Tonsillen eines Hundes injiziert hatte, bis in die mediastinalen und subpleuralen Lymphdrüsen verfolgen und illustriert damit den Weg über die MandelnLymphbahnen-Pleurakuppe-Lunge. Baup verfolgte lebende Tuberkel bazillen beim Hunde von den Tonsillen bis in das Mediastinum, Loch und auch Pertik erkennen diesen Weg an. Daß dieser von den genannten Seiten abgelehnte Modus in der Tat praktisch in Betracht kommen kann, zeigen die interessanten neuesten Versuche Kochs und Moellers, denen es gelang, Kaninchen von der Mundhöhle und den oberen Verdauungswegen aus tuberkulös zu machen. „Jedoch kam es bemerkenswerterweise nicht zu einer allgemeinen Infektion des Blutes, sondern es entwickelte sich eine hauptsächlich auf den Oberlappen und die freien Ränder der Lunge beschränkte chronisch verlaufende, der menschlichen Lungentuberkulose ähnliche Erkrankung." Plate hat darauf aufmerksam gemacht, daß es für den Verlanf einer Erkrankung, die mit Tonsillaraffektion einhergeht oder durch letztere ausgelöst wird, oft von ausschlaggebender Bedeutung ist, ob die Halslymphdrüsen von hier aus befallen werden oder nicht. Er konnte an einem Falle zeigen, daß bei der Beteiligung der Halslymphdrüsen keine Allgemeinerkrankung erfolgte, während bei demselben Individuum eine Tonsillenaffektion ohne Ubergreifen auf die Halslymphdrüsen von einer Allgemeininfektion gefolgt wurde. Es ist aber wohl denkbar, daß diese Vorstellung auch für die Tuberkuloseinfektion bis zu einem gewissen Grade Gültigkeit hat und würde dann die Erkrankung der Halslymphdrüsen einen wesentlich anderen prognostischen Wert erhalten, als im ïbrigen bislang angenommen wird. Die Mitteilung Grobers, der fand, daß bei $14,3 \%$ derjenigen Menschen, die an tuberkulösen Halslymphdrüsen operiert worden sind, später eine Lungenphthise auftrat, zeigt eine relativ geringe Neigung dieser Tuberkulose zur weiteren Ausdehnung. Es führen aber eben so unendlich viele Wege nach Rom, daß eine einseitige Auffassung der komplizierten Wirklichkeit niemals gerecht werden kann. Und so müssen wir hier auch noch einer 
weiteren Infektionsibbertragung Erwähnung tun. das ist die Cibertragung auf dem Blutwege. Wir wissen aus zahlreichen Versuchen, dals die Lokalisation der 'Tuberkulose in den Lungen auch in den Lungenspitzen keineswegs immer auf die Eintrittsstellen der Bakterien im Bronchialbaum zurïckzuführen ist, sondern auch bei Injektionen des Virus an anderen Körperstellen und in die Blutbahn erfolgen kamn (Versuche von Backmeister und anderen). So müssen wir den Übertritt von Bazillen in die Blutbahn beim Aufenthalt in den Tonsillen auch ohne histologische Verïnderung durchaus für möglich halten (A uf recht). Datür gibt die gute Blutversorgung der Follikel (Abb. 2) jedenfalls Gelegenheit. Friederici fand nach Tujektion von Karmin in das venöse Gefäß system. in die Pleura und die Bauchhöhle dasselbe nach 24 Stunden in den

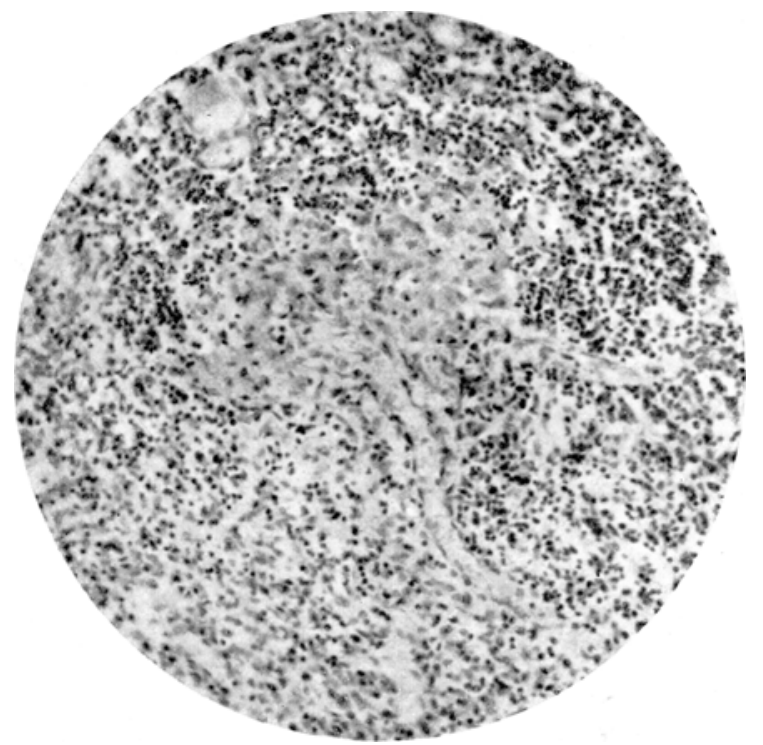

Abb. 2.

Tonsillen wieder. Der enge Konnex der Tonsillen mit dem Blutgefäßsystem wird meines Erachtens durch die von diesen ausgehenden rheunatischen Erkrunkungen eindringlich demonstriert. (Citron.)

Zusammenfassend kann die Infektion der Tonsillen durch die Atmung. durch Mund oder Nase, durch die Nahrung. dann sekundär durch Blut-oder Lymphbahnen von anderen erkrankten Körperstellen aus und durch den eigenen infektiösen Auswurf erfolgen (von Scheibner). In welchem Umfange diese Vorgänge für die Lungentuberkulose malßgebend sind, läßt sich aus den zur Zeit noch widersprechend lautenden Ansichten der Autoren nicht mit sicherheit ermessen und kamn nur durch weitere Beobachtungen und Versuche einer Klärung entgegengeführt werden.

Unsere Untersuchungen haben sich dabei lediglich auf die derzeitige Häufigkeit von tuberkulösen Veränderungen an den Tonsillen erstreckt. Solche Untersuchungen sind bereits seit geraumer Zeit systematisch erfolgt und die Ergebnisse dieser Arbeiten in gewisser Hinsicht ïberraschend, denn es mul3 auffallen, daß die Tonsillen trotz ihrer exponierten Lage bei Tuberkuloseaffek- 
tionen nur in geringem Maße miterkranken und noch viel seltener primär befallen werden, wenn auch die sekundäre Infektion schon nach früheren Untersuchungen sehr häufig ist. Eine gewisse Resistenz der Tonsillen gegen die Tuberkulose ist nun auch ganz unverkennbar und durch die relative Seltenheit, sowie die ganz auffallende Milde der Erkrankung erwiesen. Sie verhalten sich also in dieser Hinsicht wesentlich verschieden von den übrigen Lymphdrüsen des Körpers. Ich glaube mir dieses durch die gute Blutversorgung im Verein mit der besonderen Physiologie ihres lymphatischen Gewebes vollauf erklären zu können. Wood konnte diese Verhältnisse im Tierexperiment belegen. Die relative Resistenz der Gaumentonsille gegen die Etablierung manifester tuberkulöser Prozesse charakterisiert denn auch das ganze Krankheitsbild. Es kommt hei der Tonsillartuberkulose nämlich nur in den wenigsten Fällen zu einer bereits makroskopisch erkennbaren tuberkulösen Veränderung und Ulzeration des Gewebes, sondern die Organe zeigen in der übergroßen Mehrzahl der Fälle rein äußerlich gar keine Besonderheiten, wie $S$ traßmann, der als erster eine Anzahl von Fällen systematisch auf die Tonsillartuberkulose untersuchte. und alle folgenden Beobachter einstimmig feststellen und auch wir vollauf bestätigen können. Straßmann, Dmochowsky, Ito fassen die Tonsillartuberkulose als rein sekundäre Erkrankung auf, da sie dieselbe nur bei gleichzeitiger tuberkulöser Erkrankung der Atmungswege feststellen komnten. Daß die sekundäre Erkrankung hier bei weitem im Vordergrund steht, hat sich auch in allen späteren Arbeiten bestätigt, nur haben die späteren Untersuchungen doch auch die Möglichkeit einer primären bzw. gleichzeitigen Erkrankung sichergestellt. Das allgemeine Interesse wird sich natürlich in erster Linie auf die letztere Erkrankungsform richten. Lubarsch wies durch Krückmann als erster auf die Gaumenmandeln als Eingangspforte der Tuberkelbazillen hin, während Orth zuerst primäre Tonsillartuberkulose, und zwar bei nicht tuberkulösen Kindern fand. Die theoretische Möglick keit einer solchen Infektion haben wir einleitend bereits auseinandergesetzt, sie wird durch die Feststellungen Strauß, zitiert bei von S'cheibner, daß ein großer Prozentsatz von Leuten, die sich in tuberkelbazillenhaltiger Umgebung aufhalten, ohne selbst tuberkulös zu sein, virulente Bakterien im Nasen-Rachenraum beherbergen, weiter illustriert. Tatsächlich ist die sichere primäre Erkrankung der Tonsillen jedoch recht selten. Alle mir bekannten Untersuchungsreihen berichten immer nur ïber vereinzelte Fälle primärer Tonsillartuberkulose. Die Referate Aufrechts (Gördeler), der bei 47 Fällen 6 Fälle primärer Tonsillartuberkulose fand, stehen mit $12,75 \%$ bisher isoliert da. Aufrecht ist infolge seiner Beobachtungen zu der Utberzeugung gekommen, daß die Tuberkelbazillen in erster Linie durch die Tonsillen in den menschlichen Körper eintreten, von hier aus in die Blutbahn übergehen und dann vom Blutweg aus die tuberkulösen Veränderungen setzen; ihm schließen sich Beckmann, Wolff und von Wiesmayer an. Lubarsch fand in einer großen Untersuchungsreihe $1,5 \%$ ganz sichere primäre Tonsillartuberkulose, vorwiegend bei Kindern. Auch Bandelier gibt an, daß sehr wohl in den ersten Verdauungswegen, wie auch Orth sagt, eine primäre Tuberkulose auf Grund einer Inhalationsinfektion entstehen kann; er selbst fand bei der Untersuchung von 100 Tonsillenpaaren 2 Fälle von vielleicht primärer Tonsillartuberkulose, mißt im iibrigen aber 
den Tonsillen als Eingangspforten einer tuberkulösen Infektion der Lunge keine große Bedeutung zu. Ebenso konstatiert von Scheibner, wie erwähnt, die Möglichkeit einer primären Infektion der Tonsillen und fand bei 60 untersuchten Fällen 5\% mit großer Wahrscheinlichkeit primärer Tonsillartuberkulose. Weitere Berichte über primäre Tonsillartuberkulose liegen u. a. von Ritter, Friedemann und Gallois vor. Ruge führt einen Fall von Malum pottii suboccipitale auf primäre Tonsillartuberkulose zurück. Die Angaben Gottsteins beruhen, abgesehen von der Tonsillaruntersuchung auf klinischer Beobachtung und können daher in diesem Rahmen nicht verwertet werden. Aber selbst unter Berücksichtigung der Tatsache, daß mir eine Reihe diesbezüglicher Arbeiten nicht zugänglich war, sind die Beobachtungen primärer Tonsillartuberkulose gering. Einen ganz auffallend geringen Prozentsatz von Tonsillartuberkulose fand der Amerikaner Levy, der bei 486 Fällen von Larynxtuberkulose nur 5,31\% Tuberkulose des Pharynx und Larynx konstatierte. Ich kann mir das nur erklären, wenn er diesen Befund ohne histologische Nachprüfung erhoben hat. Im Tierversuch konnten, wie bereits gesagt, von Bau mgarten, Baup, Koch und Moellers die primäre Infektion der Tonsillen nachweisen. Trotz des geringen Befundes primärer manifester Tuberkulose der Tonsillen werden dieselben außer von den erwähnten Autoren noch von HoppeSeyler, Grawitz, Petruschky, Baup als Eingangspforten für die Tuberkelbazillen mit in Rechnung gezogen, wohl nicht zum mindesten auch auf Grund der latenten Tonsillartuberkulose.

Die meisten Arbeiten aber weisen ein beträchtliches Material an sekundärer Tonsillartuberkulose auf, und ohne die Ergebnisse einzeln zu berichten, möchte ich feststellen, daß in den größeren Untersuchungsreihen die sekundäre Tonsillartuberkulose mit durchschnittlich bis $\mathrm{zu} 60 \%$ angegeben wird (Kaufmann), während bei kleineren, Zufälligkeiten noch mehr ausgesetzten Beobachtungszahlen oft wohl noch ein höherer Prozentsatz herausspringen könnte, und zwar beziehen sich diese Resultate fast ausschließlich auf Fälle mit gleichzeitiger ulzeröser Lungentuberkulose. Bemerkenswert ist noch, daß die hypertrophischen Mandeln im allgemeinen keine nennenswerten Befunde an Tuberkulose ergeben (Pertik), ohne daß sie eine tuberkulöse Affektion bei mehr oder weniger großer Infektionsmöglichkeit verhindern, wie die Untersuchungen Bandeliers ergeben. Im übrigen aber sind die diesbezüglichen Befunde wenig ergiebig. Sevall fand von 772 exstirpierten Tonsillen 30 tuberkulös $=3,9 \%$, wobei aus dem mir vorliegenden Referat nicht zu ersehen ist, wie groß der Prozentsatz der Tuberkulose überhaupt bei den operierten Patienten war. Friedemann fand bei Kindern mit chronischer Hypertrophie der Tonsillen unter 54 Fällen nur einmal Tonsillartuberkulose. Es ist diese Tatsache wohl auch wieder mit der starken Hyperämie hypertrophischer Tonsillen zu erklären.

Meine Untersuchungen erstrecken sich nun auf 112 Fälle, die etwa zur Hälfte tuberkulöse Individuen betreffen, zur anderen Hälfte solche ohne makroskopisch nachweisbare Tuberkulose anderer Organe. Dabei habe ich weiter keine besonderen Gesichtspunkte in der Auswahl des Materials walten lassen, höchstens solche Fälle bevorzugt, bei denen das lymphatische Gewebe noch einigermaßen erhalten war und nicht bereits durch ausgedehnten Zerfall oder Atrophie fast geschwunden schien. Ich füge die Untersuchungsreihen an. 


\begin{tabular}{|c|c|c|c|c|c|}
\hline $\begin{array}{l}\text { Prot. } \\
1920\end{array}$ & & Alter & Geschl. & Tons. & $\begin{array}{l}\text { Drüsen- } \\
\text { Tbe. }\end{array}$ \\
\hline 121 & Tbc. pulm. bds. fortgeschritten , . . & $16 \mathrm{~J}$. & m. & 0 & + \\
\hline 133 & Status n. Appondektomie. Peritonitis & $11 \mathrm{~J}$. & m. & 0 & 0 \\
\hline 135 & $\begin{array}{l}\text { Miliartbc. der Lungen. Tbc. Bronchialdrüsen. } \\
\text { Meningitistbc. . . . . . . . . . . . }\end{array}$ & & & + & + \\
\hline 147 & Fortgeschrittene Tbc. pulm. . . . . . . . . . & $39 \mathrm{~J}$. & w. & + & + \\
\hline 152 & Fortgeschrittene Tbc. pulm. und Kchlkopftbc. & $63 \mathrm{~J}$. & m. & + & + \\
\hline 151 & Aortitis luica. . . . . . . . . . . . . & $55 \mathrm{~J}$. & w. & 0 & 0 \\
\hline 155 & Fortgeschrittene Tbc. pulm. und Kchlkopftbc. . . & $45 \mathrm{~J}$. & m. & + & $\div$ \\
\hline 160 & Tbe. Caries der Halswirbelsäule, Nierensklerose & $77 \mathrm{~J}$. & w. & $\mathbf{0}$ & 0 \\
\hline 166 & Fortgeschrittene Tbc. pulm. und Kehlkopftbc. . . & $54 \mathrm{~J}$. & m. & + & + \\
\hline 173 & Fortgeschrittene The. pulm. und Kehlkopftbe. . . & $22 \mathrm{~J}$ & m. & + & + \\
\hline 204 & $\begin{array}{l}\text { Ältere Tbe pulm mit frischer Aussaat, The. Caries } \\
\text { des Os sacrums }\end{array}$ & & $\mathrm{m}$ & & \\
\hline 210 & Influenza-Pneumonie . . . . . . . . . . . . & $24 \mathrm{~J}$ & $\mathrm{w}$. & + & + \\
\hline 212 & Influenza-Pneumonie & $21 \mathrm{~J}$ & $w$ & 0 & 0 \\
\hline 229 & Influenza-Pneumonic $\ldots \ldots \ldots \ldots$ & $22 \mathrm{~J}$. & $w$. & 0. & 0 \\
\hline 244 & Influenza-Pneumonie, Verkäøsung der Mes.-Drüsen & $17 \mathrm{~J}$. & w. & 0 & 0 \\
\hline 264 & Fortgeschrittene Tbc. pulm. und Kehlkopftbe. . . & $20 \mathrm{~J}$ & w. & + & + \\
\hline 266 & Ausgedelinte frische Tbc. pulm., tbc. Meningitis & $64 \mathrm{~J}$. & m. & 0 & + \\
\hline 270 & Tbe. pulm. Tbe. Meningitis . . . . . . . & 3 J. & w. & + & + \\
\hline 278 & Arteriosklerose. Nierensklerose . . . . . . . . . & $69 \mathrm{~J}$. & w. & + & \\
\hline 288 & Tbc. pulm. mit frischer Aussaat . . . . . . & $26 \mathrm{~J}$. & w. & + & \\
\hline 356 & Fortgeschrittene Tbc. pulm. ......... & $19 \mathrm{~J}$. & w. & + & + \\
\hline 357 & Viridanssepsis, Endocarditis ulcerosa aortae. & $39 \mathrm{~J}$. & m. & 0 & 0 \\
\hline 327 & Rippentbo. Empyem . . . . . . . . . . . . . & $17 \mathrm{~J}$. & m. & + & + \\
\hline 367 & Ältere und frische Tbc. pulm. Meningitis tbc. . & $24 \mathrm{~J}$. & w. & + & + \\
\hline 370 & Influenza-Pneumonie, Pleuritis $\ldots \ldots$ & $28 \mathrm{~J}$. & w. & 0 & + \\
\hline 775 & Sehwielenbildung der rechten Spitze, Tuberkel in den & & & & \\
\hline & Mediastinaldrüsen . . . . . . . . . . . & $14 \mathrm{~J}$. & m. & + & + \\
\hline 391 & Bronchopneumonie. Myocarditis postdiphtherica & 6 Mt. & - & 0 & + \\
\hline 393 & Fortgesohrittene Tbc. pulm. und Kehlkopftbe. . & - & - & + & + \\
\hline 399 & Diphtherie, Bronchial- und Mesenterialdrüsentbe. & $5 \mathrm{~J}$. & w. & 0 & + \\
\hline 402 & Fortgesshrittene The. pulm. und Kellkopftbc. . . & $46 \mathrm{~J}$. & m. & + & + \\
\hline 409 & Bronchopneumonie . . . . . . . & 4 Mt. & - & 0 & 0 \\
\hline 417 & Tbc. pulm. mit miliarer Aussaat . . . . . & $1 \mathrm{~J}$. & - & + & + \\
\hline 419 & Chron. Pneumonie. Endocarditis verrucosa, Hilus- & & & & \\
\hline & 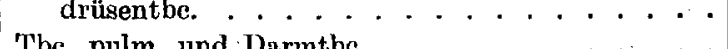 & $17 \mathrm{~J}$. & w. & + & + \\
\hline 420 & 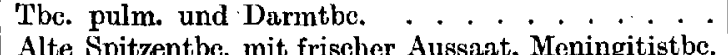 & 34 J. & w. & + & + \\
\hline 421 & Alte Spitzentbe. mit frischer Aussaat, Meningitistbe. & $8 \mathrm{~J}$. & w. & + & + \\
\hline 422 & Tbe. pulm. und Drüsentbc. Tbc. Darmgeschwüre . & 9 Mt. & $\mathbf{m}$. & + & + \\
\hline 430 & $\begin{array}{l}\text { Drüsentbc., Bronchialdrüsentbc., Mesenterialkonglo- } \\
\text { merat. Tuberkel in der rechten Spitze. Tbc. }\end{array}$ & & & & \\
\hline & Peritonitis . . . . . . . . . . . . & $22 \mathrm{~J}$. & m. & + & + \\
\hline 435 & The. pulm. mit Kavernenbildung . & $43 \mathrm{~J}$. & m. & + & + \\
\hline 438 & Mitralinsuffizienz und Aortenstenose & $29 \mathrm{~J}$. & m. & 0 & 0 \\
\hline 441 & Sepsis. Nackenphlegmone ......... & $52 \mathrm{~J}$. & m. & 0 & + \\
\hline 444 & Lungentbc. Darmtbe. Beg. Kavernenbildung . & $21 \mathrm{~J}$. & w. & + & $\div$ \\
\hline 445 & Fortgeschrittene Tbc. pulm. . . . . . . . . & $21 \mathrm{~J}$. & w. & + & $+t$ \\
\hline 450 & Maligne Sklerose....... & $36 \mathrm{~J}$. & m. & 0 & 0 \\
\hline 451 & Morbus Addison, isolierte Nebennierentbc. . . & $19 \mathrm{~J}$. & w. & 0 & 0 \\
\hline 453 & Fortgesehrittene Tbc. pulm. und Kehlkopftbc. . & $42 J$. & m. & + & + \\
\hline
\end{tabular}




\begin{tabular}{|c|c|c|c|c|c|}
\hline $\begin{array}{l}\text { Prot. } \\
1920\end{array}$ & & Alter & Geschl. & $\begin{array}{l}\text { Tons. } \\
\text { Tbe. }\end{array}$ & $\begin{array}{l}\text { Drüsen- } \\
\text { Thoc. }\end{array}$ \\
\hline 455 & Kruppöse Pneumonie . . . . . . . . . . & $1 \mathrm{~J}$. & $\mathbf{m}$. & 0 & 0 \\
\hline 457 & Karzinose . . . . . . . . . . & $54 \mathrm{~J}$ & w. & 0 & 0 \\
\hline 458 & Laennecsche Leberzirrhose . . . . . . . . . & $48 \mathrm{~J}$. & w. & 0 & 0 \\
\hline 459 & Fortgeschritte Tbe. pulm. . . . . . . . & $29 \mathrm{~J}$. & $\mathrm{m}$. & + & + \\
\hline 460 & Ösophaguskarzinom $\ldots \ldots \ldots$ & $66 \mathrm{~J}$. & m. & 0 & 0 \\
\hline 461 & Fortgeschrittene The pulm. . . . . . . . . & $23 \cdot J$ & $\mathrm{~m}$. & + & + \\
\hline 466 & Ca. pylori . . . . . . . & $66 \mathrm{~J}$. & $\mathrm{m}$. & 0 & 0 \\
\hline 469 & Aortitis luica. . . . . . . & $41 \mathrm{~J}$. & $\mathrm{m}$. & 0 & + \\
\hline 472 & Sepsis. Oberlippenphlegmone , . . . & $29 \mathrm{~J}$. & m. & 0 & 0 \\
\hline 473 & Endocarditis verr. Mitralinsuffizienz .. . & $18 \mathrm{~J}$. & $\mathrm{m}$. & 0 & 0 \\
\hline 474 & Diphtherie. . . . . . . . . . & $11 \mathrm{M}$. & m. & 0 & 0 \\
\hline 475 & Sepsis post abortum . . . . & $37 \mathrm{~J}$. & w. & 0 & 0 \\
\hline 476 & Tbc, pulm. mit Kavernenbildung & $18 \mathrm{~J}$. & w. & + & $t$ \\
\hline 477 & Appendizitis. Peritonitis . . . & $63 \mathrm{~J}$ & $\mathrm{~m}$. & 0 & 0 \\
\hline 478 & Bronchopneumonie . . . . . . . . & $\mathrm{I}^{1 / 4} \mathrm{~J}$ & m. & 0 & 0 \\
\hline 479 & $\begin{array}{l}\text { Mediastinal- und Mesenterialdrüsentbc. Vom Hilus } \\
\text { ausgehende Lungentbc. mit beg. Verküsung. } \\
\text { Tbc. Meningitis. Tbc. Darmgeschwüre. }\end{array}$ & & & & \\
\hline 481 & $\begin{array}{l}\text { Tbe- Meningitis. Tbe. Darmgeschwüre. . . . } \\
\text { Gliom der Pons cerebri . . . . . . . . . . }\end{array}$ & $6 \mathrm{~J}$ & w. & + & $+\frac{1}{0}$ \\
\hline 483 & Fortgeschrittene altere und frische Tbc pulm. und & $16 \mathrm{~J}$. & w. & 0 & \\
\hline & Kehlkopftbc. . . . . . . . . . . . & $63 \mathrm{~J}$. & m. & + & + \\
\hline 484 & Karn. Pneumonie. Meningitis seropurulenta. & 3 Mt. & m. & 0 & 0 \\
\hline 485 & Alte Spitzentbc. Pericarditis tbc. . . ... & $62 \mathrm{~J}$. & m. & 0 & $\div$ \\
\hline 487 & Fortgeschrittene Tbe. pulm. und Kehlkopftbe. . & $33 \mathrm{~J}$. & $\mathrm{m}$. & + & + \\
\hline 489 & Aortenaneurysma. Maligne Sklerose . . . . . . & $64 \mathrm{~J}$ & m. & 0 & 0 \\
\hline 490 & Eingeklemmte Hernie, Peritonitis . . . . . & $74 \mathrm{~J}$. & m. & 0 & 0 \\
\hline 492 & Fortgeschrittene Tbc. pulm. und Kehlkopftbc. & $53 \mathrm{~J}$. & m. & + & + \\
\hline 493 & Bronchopneumonie . . . . . . . . . & $51 / 2 \mathrm{Mt}$ & m. & 0 & 0 . \\
\hline 496 & $\begin{array}{l}\text { Hilusdrüsentbe, frische Aussaat in den Lungen, } \\
\text { Miliartbc. }\end{array}$ & $21 \mathrm{~J}$. & w. & + & + \\
\hline 497 & Bronchopneumonie. Pleuritis . . . . . . . . & $57 \mathrm{~J}$. & w. & 0 & 0 \\
\hline 501 & Ösophaguskarzinom . . . . . . . . . . . & $61 \mathrm{~J}$. & $\mathrm{m}$. & 0 & 0 \\
\hline 504 & $\begin{array}{l}\text { Status n. Amputation des rechten Unterschenkels. } \\
\text { Sepsis }\end{array}$ & $59 \mathrm{~J}$. & m. & 0 & 0 \\
\hline 506 & $\begin{array}{l}\text { Caries der Rippen und Halswirbel Alte Spitzen- } \\
\text { narben, frische Aussaat im Unterlappen }\end{array}$ & - & - & 0 & \\
\hline 505 & $\begin{array}{l}\text { Mesenterialdrüsentbe. Tbc. Darmgesehwüre. Kein } \\
\text { Lungenbefund }\end{array}$ & 21. J. & n., & 0 & 0 \\
\hline 510 & Starke Bronchiektasenbildung. Amyloidnephrose . & $69 \mathrm{~J}$. & $\mathrm{m}$. & + & + \\
\hline 513 & Schädelbruch. . . . . . . . . . . & $31 \mathrm{~J}$. & w. & 0 & + \\
\hline 517 & Mesenterialdrüsentbc. Meningitis purulenta . . . . & $5 \mathrm{~J}$. & w. & + & 0 \\
\hline 518 & Osteomyelitis tibiae, Sepsis . . . . . . . & $9 \mathrm{~J}$. & m. & 0 & 0 \\
\hline 519 & Tbc. pulm. mit Kavernenbildung . . . . . . & $53 \mathrm{~J}$. & w. & + & + \\
\hline 520 & $\begin{array}{l}\text { Hilusdrüsentbc. Solitärtuberkel und frische Aus- } \\
\text { saat im rechten Unterlappen. Tbe. Meningitis }\end{array}$ & & & & \\
\hline 523 & Pneumonie. Pleuritis . . . . . . . . . . & $8 \mathrm{Mt}$. & w. & 0 & 0 \\
\hline 532 & Bronchopneumonie : . & $11 / 2 J$ & m. & 0 & 0 \\
\hline 534 & Endocarditis ulcerosa. & $39 \mathrm{~J}$. & m. & 0 & $\mathbf{0}$ \\
\hline 535 & Ca. des Rektums . . . . . . . . . . . . . & $63 \mathrm{~J}$. & w. & 0 & 0 \\
\hline 536 & $\begin{array}{l}\text { Schwere ulzeröse Endokarditis der Mitralis und } \\
\text { Aorta, subchron. embolische Herdnephritis }\end{array}$ & & & & \\
\hline 538 & $\begin{array}{l}\text { Aorta, subchron. embolische Herdnephritis } \\
\text { Ca. des Ductus cysticus und hepaticus . . . . }\end{array}$ & $52 \mathrm{~J}$. & $\begin{array}{l}\text { m. } \\
\text { w. }\end{array}$ & 0 & 0 \\
\hline
\end{tabular}




\begin{tabular}{|c|c|c|c|c|c|}
\hline $\begin{array}{l}\text { Prot. } \\
1920\end{array}$ & & Aiter & Geschl. & $\begin{array}{l}\text { Tons. } \\
\text { Tbe. }\end{array}$ & $\begin{array}{l}\text { Drüsen- } \\
\text { Tbc. }\end{array}$ \\
\hline 539 & $\begin{array}{l}\text { Endocarditis ulcerosa, Aortitis. Subchron. Glome- } \\
\text { rulonephritis (Remission). }\end{array}$ & & & & \\
\hline 542 & Starke Arteriosklerose und Koronarsklerose. Nieren- & $47 \%$ & $\mathrm{~m}$. & 0 & 0 \\
\hline & sklerose. . . . . . . . . . . & $74 \mathrm{~J}$. & $m$. & 0 & 0 \\
\hline 543 & Frische Tbc. pulm. bds. mit beg. Verkäsung & $35 \mathrm{~J}$. & w. & $\mathbf{0}$ & 0 \\
\hline $\mathbf{5 4 5}$ & $\begin{array}{l}\text { Status nach Exartikulation des rechten Ober- } \\
\text { schenkels wegen Coxitis tbc. Eben sichtbare }\end{array}$ & & & & \\
\hline 546 & $\begin{array}{l}\text { Tuberkelherde im rechten Oberlappen..... } \\
\text { Ca. der Blase. Bronchopneumonie. . . . . . }\end{array}$ & $21 \mathrm{~J}$. & m. & $\mathbf{0}$ & 0 \\
\hline 554 & $\begin{array}{l}\text { Ca. der Blase. Bronchopneumonie . . . } \\
\text { Tbe. pulm., Hilusdrüsentbc., tbe. Meningitis }\end{array}$ & $76 \mathrm{~J}$. & w. & $\mathbf{0}$ & $\div$ \\
\hline 556 & $\begin{array}{l}\text { Tbe. pulm., Hilusdrüsentbc., tbe. Meningitis . . } \\
\text { Fortgeschrittene Tbc. pulm. . . . . . . }\end{array}$ & $11 / 2 \mathrm{~J}$ & m. & + & + \\
\hline$\tilde{5} 61$ & 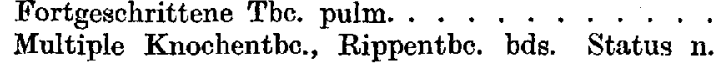 & $31 \mathrm{~J}$. & m. & + & $\div$ \\
\hline & Resektion der V. linken Rippe. Tbc. Pleuritis . & $69 \mathrm{~J}$. & $\mathrm{m}$ & 0 & + \\
\hline 566 & Tbc. pulm. mit Kavernenbildung . . . . . . . & $30 \mathrm{~J}$. & $\mathrm{m}$. & + & $\div$ \\
\hline 576 & Appendizitis. Peritonitis . . . & $31 \mathrm{~J}$. & $w$. & + & + \\
\hline 568 & Tbo. pulm. et laryngis . . . . . & $32 \mathrm{~J}$. & m. & + & + \\
\hline 577 & Bronchopneumonie, Verkäste Mediastinaldrüsen . & $1 \mathrm{~J}$. & w. & 0 & + \\
\hline 581 & Septische Thrombophlebitis . . . . . . & $66 \mathrm{~J}$. & $\mathrm{m}$. & + & + \\
\hline 599 & The. pulm. et laryngis . . . . . . . & $33 \mathrm{~J}$. & m. & + & $\div$ \\
\hline 606 & Fortgeschrittene The. pulm. und Tbc. laryngis & $43 \mathrm{~J}$. & m. & + & +- \\
\hline 619 & Tbe. pulm. et laryngis . . . . . & $39 \mathrm{~J}$. & m. & + & + \\
\hline 645 & The. pulm. frisch, Meningitis tbe. & $7 \mathrm{Mt}$. & $\mathrm{m}$. & + & + \\
\hline 657 & Primäre Darmtbc. Meningitis tbe. . . . . . . & $1 \mathrm{~J}$. & w. & + & + \\
\hline 662 & Alter Kreideherd mit frischer Aussaat. The. Me- & & & & \\
\hline & ningitis . . . . . . . . . . & $42 \mathrm{~J}$. & m. & 0 & 0 \\
\hline 664 & Tbe. pulm. et laryngis . . . . & $46 \mathrm{~J}$ & $w$. & + & + \\
\hline 666 & Tbc. pulm. mit Kavernenbildung & $20 \mathrm{~J}$. & w. & + & $\div$ \\
\hline 671 & Nicht ulz. The. pulm. . . . . & $67 \mathrm{~J}$. & w. & 0 & 0 \\
\hline 691 & Tbe. pulm. . . . . . & $5 \mathrm{~J}$. & w. & 0 & + \\
\hline 694 & Tbc. pulm. & $29 \mathrm{~J}$. & $\mathrm{w}$. & + & $\div$ \\
\hline
\end{tabular}

Außerdem wurden 34 Fälle operativ gewonnener Tonsillen bzw. Rachenmandeln untersucht.

Wenn wir diese Resultate in die wesentlichen zusammengehörigen Untergruppen zusammenfassen, so finden wir folgendes:

38 Fälle fortgeschrittener Lungentuberkulose zeitigten 2 Fälle ohne Tonsillartuberkulose $=94,7 \%$ Tonsillartuberkulose.

13 Fälle nicht ulzerierter Lungentuberkulose zeitigten 8 Fälle Tonsillartuberkulose $=53,2 \%$ Tonsillartuberkulose.

9 Fälle von Tuberkulose ohne Beteiligung der Lungen zeitigten 3 Fälle von Tonsillartuberkulose $=33,3 \%$ Tonsillartuberkulose.

48 Fälle ohne makroskopischen aktiven Tuberkulosebefund zeitigten 6 Fälle von Tonsillartuberkulose $=12,5 \%$ primärer Tonsillartuberkulose.

34 Fälle hypertrophischer Tonsillen bzw. Rachenmandeln zeitigten nur 1 Fall von Tonsillartuberkulose.

Wir finden also eine ganz auffallende Zunahme der Tonsillartuberkulose in allen Gruppen. Die primäre Tonsillartuberkulose zeigt mit $12,5 \%$ neben 
dem von Aufrecht gegebenen Prozentsatz etwa das Doppelte der übrigen bisherigen Resultate; dabei habe ich als primäre Tonsillartuberkulose auch die Fälle verwertet, bei denen sich außer an den Tonsillen nur noch tuberkulöse Veränderungen vereinzelter Hilus- oder Bronchialdrüsen zeigten. Naegeli spricht bei deutlich deszendierender Lymphdrüsenaffektion, trotz des unbedeutenden Tonsillarbefundes, diesen als primären Herd an. Die hier in Betracht kommenden Fälle lassen einen Zweifel wegen der Geringfügigkeit und Getrenntheit der Drüsenerkrankungen gar nicht aufkommen. Im übrigen habe ich als Kriterium der Diagnose Tuberkulose nur ausgebildete Epitheloidtuberkel mit oder ohne Langhanssche Riesenzellen betrachtet, ohne die häufig sehr verdächtigen Follikel mit beginnender Hyalinisierung und Aufquellung der Zellen oder narbige Prozesse mit in Rechnung zu ziehen. In der übergroßen Mehrzahl der Fälle handelt es sich dabei um streng lokalisierte, mehr weniger frische Tuberkelbildung, die oft so isolierte Herdchen bilden, daß bei einer ganz genauen Durchsuchung der Organe (ich habe meist nur 5-10 Schnitte aus beiden Tonsillen untersucht) sich wohl noch eine Reihe positiver Fälle mehr hätte finden lassen. Besonders bemerkenswert erscheint mir noch die erhebliche Beteiligung der Tonsillen mit manifesten, tuberkulösen Prozessen bei Fällen, in denen eine Sputuminfektion so gut wie ausgeschlossen ist. Die 15 Fälle nicht ulzerierter Tuberkulose waren klinisch ohne Bazillenbefund gewesen. Aber auch die sicher sekundären Erkrankungen zeigen eine bisher unbekannte Häufigkeit von $94,7 \%$. Im Gegensatz zu der erheblichen Zunahme der Tonsillartuberkulose im ganzen stehen nun die Befunde an den operierten, hypertrophischen Tonsillen und Rachenmandeln, die übrigens bis auf den einen positiven Fall sämtlich von klinisch nicht tuberkulösen Patienten stammen, bei denen wir so gut wie gar keine tuberkulöse Veränderungen feststellen konnten. Das Material wurde mir gütigst von der Ohrenabteilung des Krankenhauses, Oberarzt Dr. Sinell und seinem Assistenten Dr. Groth, überwiesen.

Wir sehen, daß sich also bezüglich der hypertrophischen Organe die Verhältnisse durch den Krieg nicht geändert haben. Dabei müssen wir wiederum die Hyperämie als den besten Schutz gegen die tuberkulöse Infektion bezeichnen.

Die ganz auffallende Zunahme der Tonsillartuberkulose möchte ich in erster Linie als einen erneuten Beweis der sinkenden Resistenzfähigkeit des Organismus gegen die Ausbreitung tuberkulöser Prozesse auswerten, ohne daraus auf die Bedeutung der Tonsillen als Eingangspforten der Tuberkelbazillen wesentlich neue Schlüsse zu ziehen. Ich glaube vielmehr, daß die herrschende Ansicht, die den Tonsillen eine gewisse Bedeutung für die Tuberkuloseinfektion nicht abspricht, ohne besonderes Gewicht auf diesen Weg zu legen, am meisten Berechtigung hat.

\section{Literatur.}

1. A uf recht, Die Genese der Lungenphthise und die Verschiedenheit der mit dem Namen ,Tuberkel" bezeichneten Gebilde. Deutsch. Arch. f. klin. Med. 75.

2. Backmeister, Aerogene oder hämatogene Entstehung der Lungenspitzenphthise. Deutsche med. Wochenschr. 1913. Nr. 24.

3. Bandelier, Die Tonsillen als Eingangspforten der Tuberkelbazillen. Brauers Beițr. z. Klin. d. Tuberkul. 6, L. 1 . 
4. Bandellier und Röpke, Die Klinik der Tuberkulose. 1920.

5. v. Baumgarten, Welche Ansteckungsweise spielt bei der Tuberkulose des Menschen die wichtigste Rolle? Deutsche med. Wochenschr. 1909. Nr. 40.

6. - Über das Verhalten der Tuberkelbazillen an den Eingangspforten der Infektion. Berl. klin. Wochenschr. 1905. Nr. 42.

7. Baup, Breitung, Die Bedeutung der oberen Luftwege als Eintrittspforten der Tuberkulose. Samml. zwanglose Abhandl. a. d. Geb. d. Nasen-, Ohren-, Mundund Halskrankheiten 4, 1901. Nr. 7.

8. Bartel, Tuberkuloseinfektion im Säuglingsalterdes Meerschweinchens und Kaninchens. Wien. klin. Wochenschr. 1905. Nr. 44.

9. - Der Gang der natürlichen Tuberkuloseinfektion beim jungen Meerschweinchen. Wien. klin. Wochenschr. 1906. Nr. 2.

10. - Die Infektionswege bei der Fütterungstuberkulose. Wien. klin. Wochenschr. 1905. Nr. 7.

11. - und Speiler, Der Gang der natürlichen Tuberkuloseinfektion beim jungen Meerschweinchen. Wien. klin. Wochenschr. 1905. Nr. 9.

12. Barth, Utber die Physiologie der Tonsillen und die Indikation zu ihrer Abtragung. Deutsche med. Wochenschr. 1907. Nr. 49.

13. Behla, Der Verlauf der Gesamttuberkulose-Mortalitätsstatistik in Preußen seit 1876 nach Altersklassen und die spezialisierte Kindertuberkulosestatistik. Berl. klin. Woohenschr. 1913. Nr. 42.

14. v. Behring, Über Tuberkuloșe. Verein f. innere Med. Berlin. Münch. med. Wochenschrift 1904. Nr. 4.

15. - Über alimentäre Tuberkulose-Infektionen im Säuglingsalter. Brauers Beitr. III.

16. - Leitsätze betreffend Phthiseogenese bei Menschen und Tieren.

17. Beitzke, Über die Häufigkeit der Tuberkulose am Leichenmaterial des Berliner pathologischen Instituts. Berl. klin. Wochenschr. 1909. Nr. 9.

18. - Untersuchungen über die Infektionswege der Tuberkulose. Virehows Areh. 210.

19. - Über den Weg der Tuberkelbazillen von der Mund - und Rachenhöhle zu den Lungen, mit besonderer Berücksichtigung der Verhältnisse beim Kinde. Virchows Arch. 184.

20. Bessau, Über die biologischen Vorgänge bei der Tuberkulinbehandlung. Münch. med. Wochenschr. 1915. Nr. 10.

21. Bliedung, Greifswald, Die Einwirkung der Kriegsernährung auf die Lungentuberkulose. Brauers Beitr. z. Klin. d. Tuberkul. 43, Heft 3/4.

22. Braun, E., Die Häufigkeit der Miliartuberkulose im Greisenalter. Korrespondenzbl. f. Schweiz. Ärzte 47, 1917, H. 35.

23. Burkhardt, Über Häufigkeit und Ursache mensehlicher Tuberkulose auf Grund von ca. 1400 Sektionen. Zeitschr. f. Hyg. 53.

24. Dietl, Grundlagen der Tuberkulosepathologie. Wien. med. Wochensehr. 1919. Nr. 32.

25. D mochowsky, Über sekundäre Erkrankungen der Mandeln und Balgdrüsen an der Lungenwurzel bei Schwindsüchtigen. Zieglers Beitr. 10, 481.

26. Dresen, Beitrag zur Statistik der Tuberkulosesterblichkeit in Baden. Deutsche med. Wochensehr. 1913. Nr. 22.

27. Drügg, Unterernährung und chirurgische Tuberkulose. Deutsche med. Wochenschr. 1919. 376.

28. Eisner, Wolf, Berlin, Über Zusammenhänge zwischen tuberkulöser Infektion und den konstitutionellen Diathesen (exsudative Diathese, Spasmophilie usw.). Münch. med. Wochenschr. 1920. S. 93.

29. Franz, Kriegstuberkulose und Tuberkulosekrieg. Wien. med. Wochenschr. 1916. Nr. 18.

30. Friedmann, Über die Bedeutung der Gaumentonsillen von jungen Kindern als Eingangspforte für die tuberkulöse Infektion. Zieglers Beitr. 28.

31. Gehrels, Die Mesenterialdrüsentuberkulose. Deutsche med. Wochenschr. 1919. 1128.

32. Gerhardt, Über Tuberkulose. Münch. med. Wochenschr. 1918. Nr. 21.

33. Gerwie ner, Stadt und Land, ein Problem in der Statistik und Klinik der Lungentuberkulose. Brauers Beitr. 1919. 41. 
34. Gottstein, Pharynx und Gaumentonsille, primäre Eingangspforten der Tuberkulose. Berl, kin. Woehenschr, 1896. Nr. 31 u. 32.

35. Grawitz, Die Eintrittspforten der Tuberkelbazillen und ihre Lokalisation beim Menschen. Deutsche med. Wochenschr. 1901. Nr. 41.

36. Gruber, Die Tonsillen als Eintrittspforten für Krankheitserreger, besonders für die Tuberkelbazillen. Klin. Jahrb. 14, 1905. Heft 6. Referat in Zieglers Beitr. 16.

37. - Die Infektionswege der Pleura. Deutsch. Areh. f. klin. Med. 68.

38. - Zur Tuberkulosemortalität wăhend des Krieges. Münch. med. Wodhenschr. 1919. 1260.

39. Mamburger, Über tuberkulöse Exazerbation. Wien. klin. Wochenschr. 1911. Nr. 24.

40. - Die Tuberkuloser als Kinderkrankheit. Münch. med. Wochenschr. 1908. Nr. 52.

41. - Franz, Die Tuberkulose des Kindesalters. 2. vermehrte Aufl. 1912. Ref. Zieglers Zentralbl. 25, 332.

42. - Uther die Tuberkuloseinfektion. Wien. klin. Wochenschr. 1918. Nr. 23.

43. - Tuberkulose der Kinder. Brauer-Schroeders Handb. d. Tuberkul. 1915. ō.

44. H a mel, Über den Anstieg der Tuberkulose während des Krieges. XXIII. Generalversammiung des deutschen Zentraikomitees zur Bekämpfung der Tuberizulose. Ref. Deutsche med. Wochenschr. 1919. Nr. 759.

45. v. Hayek, H., Das Tuberkuloseproblem. Springer, Berlin 1920.

46. - Innsbruck, Gesetzmäßigkeiten im Verlauf der Tuberkulose. Münch. med. Wochenschrift 1919. $1316 \mathrm{u}, 1352$.

47. Hart, Die tuberkulöse Lungenphthise alter Leute. Berl. klin. Wochenschr. 1911. Nr. 24.

48. Hellmann, Torsten Johnson, Das Verhalten der Lymphdrüsen bei Krebs, Tuberkulose und Anthrakose, sowie ihre Bedeutung als Schutzorgan im allgemeinen. Upsala Lakareförenings Förhandlingar 1909. 24, 57. Ref. Intern. Zentralbl. f. Tuberk.-Fortschr. 13, 9. 219.

49. Fenke, Neue experimentelle Feststelingen über die physiologische Bedeutung der Tonsillen. Arch. f. Laryngol. 28.

50. - Über die physiologische Bedeutung der Tonsillen. Arch. f. Laryngal. 1914.

5L. Hoppe-Seyler, Die Tuberkulose in Greisenalter. Brauer-Schroeders Handb. d. Tuberkul. 1915. 5.

52. Ibrahim, Die Säuglingstuberkulose im Lichte älterer und neuerer Forschungen. Brauers Beitr. 4.

53. Sukehiko, Ito, Japan, Über primäre Darm- und Gaumentonsillentuberkulose. Berl. klin. Wochenschr. 1904. Nr. 2.

54. de Jager, Sterblichkeitsstatistik und Ursache der Tuberkulose. Weekbl. voor Geneesk. Nr. 6. Ref. Deutsche med. Wochenschr. 1905, 1368.

55. KeBler, Über die zunehmende Häufigkeit der v. Pirquetschen Reaktion ira Kindes. alter während des Krieges. Beitr. z. Klin. d. Tuberkul, von Brauer. 44, Heft 2.

56. Kirchner, Generalversammlung des Deutschen Zentralkomitees zur Errichtung von Heilstïtten für Lungenkrankheiten, Berlin B, Mri 1906. Ref. Deutsche med. Wochenschr. 1906. 1063.

57. Kleinschmidt, Uber latente Tuberkulose im Kindesalter. Deutsche med. Wochenschrift 1914. 1120.

58. Koch, Robert, Epidemiologie der Tuberkulose. Zeitschr. f. Hyg. 67, 1910.

59. - und Moellers, Zur Frage der Infektionswege der Tuberkulose. Deutsche med. Wochenschr. 1920. Nr. 33.

60. Kraemer, Die Häufigkeit der Tuberkulose des Menschen nach den Ergebnissen von Leichenuntersuchungen und Tuberkulinprüfungen und ihre Bedeutung für die Therapie. Zeitschr. f. Hyg. u. Infekt.-Krankh. 50, 265.

61. Krause, Der Einflul der hereditiren Belastung auf Form unt Verlauf der Tuberkulose der Kinder. Arch. f. Kimderheilk. 66, 1916. Heft 1/2.

62. Kraus, Berlin, Bekämpfung der Tuberkulose. Zeitschr. f. ärztl. Fortb. 1918. 505.

63. Kretz, Spitzentuberkulose und Thorax phthisicus. Wien. klin. Wochenschr. 1918. Nr. I4. 
64. Krückmann, Über die Beziehungen der Tuberkulose und Halslymphdrüsen zu der der Tonsillen. Virchows Arch. 138.

65. Leschke, Weitere Erfahrungen über Tuberkulose im Kriege. Zeitschr. f. Tuberk. 27.

66. Levy, Tuberkulose der Tonsillen. Journ. of Americ. Assoc. 1910.

67. Lindées, Untersuchung über den Einfluß von Notjahren auf die Tuberkulosehäufigkeit, sowie die Latenz der Tuberkulose auf Grundlage der Kassationen beim finnischen Militär. Wien. klin. Wochenschr. 1909. Nr. 42.

68. Loch, Bemerkungen zum Tonsillenproblem. Arch. f. Ohren-, Nasen- und Kehlkopfheilkunde 1920. 106.

69. Lubarsch, Zur vergleichenden Pathologie der Tuberkulose. Deutsche med. Wochenschrift 1908. 1921.

70. - Über Entstehungsweise, Infektions- und Verbreitungswege der Tuberkulose. Zeitschr. f. ärztl. Fortb. 1918. A. 35.

71. - Beiträge zur Pathologie der Tuberkulose. Virchows Arch. 213.

72. Mann, Über den Einfluß der Tonsillotomie und radikalen Rachenmandeloperation auf skrofulöse Erscheinungen. Arch. f. Laryngol. 33.

73. Most, Topographie des Lymphgefäßapparates des menschlichen Körpers und ihre Beziehungen zu den Infektionswegen der Tuberkulose. Bibliotheca medica, Abt. 1. Heft 21. Ref. Ribbert, Deutsche med. Wochenschr. 1910. 230.

74. Müller, Untersuchungen über statische und dynamische Immunität bei Tuberkulose. Brauers Beitr. z. Klin. d. Tuberkul. 36, Heft 3.

75. - Tuberkulose in Göttingen. Med. Gesellsch. Göttingen. Ref.Deutsche med. Wochenschrift 1910. 1639

76. Nägeli, Ửber Häufigkeit, Lokalisation und Ausbreitung der Tuberkulose. Virchows Arch. 167.

77. Necker, Wien, Über die Häufigkeit der tuberkulösen Veränderungen am Leichenmaterial. Verhandl. d. Deutsch. Patholog. Gesellsch. VIII. Tagung 1904. Nr. 2. 129.

78. Neumann, Die durch die Kriegsverhältnisse gesteigerte Tuberkulosesterblichkeit vom Standpunkt der Rassehygiene. Deutsche med. Wochenschr. 1920. Nr. 17.

79. Noth mann, Häufigkeit der Tuberkulose im Kindesalter. Berl. klin. Wochensehr. 1910, Nr. 9.

80. Orth, Über einige Tuberkulosefragen. Berl. klin. Wochenschr. 1918. 70.

81. Pertik, Pathologie der Tuberkulose. Lubarsch und Ostertag, VIII. Jahrg., 2. Abt.

82. Pick, Die Bekämpfung der Tuberkulose in Stadt und Bezirk Aussig. Osterreich. Tuberkulosefürsorgebl. 1917. Nr. 4.

83. Piper, Über den Einfluß innerer Lebensbedingungen auf die Verbreitung der Tuberkulose auf Grund von Erfahrungen in einem Sammellazarett für tuberkulöse Kriegsgefangene. Diss. Breslau 1918.

84. Plate, Arthritiden, rheumatische Erkrankungen und ihre militärärztliche Beurteilung. Jahrb. d. Hamburg. Staatskrankenanstalten. Beiheft 1917.

85. Prey $\beta$, Über den Einfluß der Verdünnung und der künstlich erzeugten Disposition auf die Wirkung des inhalierten tuberkulösen Giftes. Münch. med. Wochenschr. 1891. Nr. 24 u. 25.

86. Prinzing, Sterbefälle und Todesursachen in Deutschland 1914-1916. Deutsche med. Wochenschr. 1920. Nr. 14/15.

87. Ranke, Die Tuberkulose der verschiedenen Lebensalter. Münch. med. Wochenschr. 1913: Nr. 38.

88. - Die Tuberkulosebekämpfung nach dem Kriege. Münch. med. Wochenschr. 1918. Nr. 12.

89. Reiche, Zur Entstehung und zum Verlauf der Lungentuberkulose im Kriege. Münch. med. Wochenschr. 1920. Nr. 5 .

90. - Reinfektion und Immunität bei Tuberkulose. Med. Klin. 1916. Nr. 40.

91. Reinhart, Anatomische Untersuchungen über die Häufigkeit der Tuberkulose. Korrespondenzbl. f. Schweiz. Ärzte 1917. 1152.

92. Réthi, Die latente Tuberkulose der Rachenmandeln. Wien. klin. Rundsch. 1900. 509. Zit. bei Pertik, Lubarsch und Ostertag. 8. 
93. Ribbert, Über primäre Tuberkulose und über die Anthrakose der Lungen und der Bronchialdrüsen. Deutsche med. Wochenschr. 1906. Nr. 30.

94. Ritter, Tuberkulosestatistik. Berl. klin. Wochenschr. 1912. 38.

95. Ruge, Die Tuberkulose der Tonsillen vom klinischen Standpunkt. Virchows Arch. 144.

96. Sajet, Tuberkulosesterfte in Nederland gedurende den mobilisatei tijd. Nederl. Tijdschrift, Geneesk. 9. Juni 1917. Ref. Intern. Zentralbl. f. Tuberkul.-Forsch. 13.

97. Sal tet, Sterbestatistik der Tuberkulose in Holland. Tijdschr. voor Geneesk. 23. Juni 1917. Ref. Deutsche med. Wochenschr. 1917. 1179.

98. Samson, Vorschläge zur Tuberkulosebekämpfung nach dem Kriege in Frankreich. Berl klin. Wochenschr. 1919. 1065.

99. Selter, Reinfektion und Immunität bei Tuberkulose. Deutsche med. Wochenschr. 1916. Nr. 10.

100. Sewall, Tuberkulose der Tonsillen. Journ. of Amer. Assoc. 9. IX. 1911.

101. Seiler, Die Tuberkulose: Nach der Todesursachen-, Erkrankungs- und Versicherungsstatistik und ihre Bedeutung für die Volkswirtschaft. 1916.

102. Selter, Die tuberkulöse Infektion im Kindesalter und ihre Bedeutung für die Phthise. Deutsche med. Wochenschr. 1918. 791.

103. Sieveking, Häufigkeit der Tuberkulosesterblichkeit im Kindesalter in Hamburg $1906-1915$.

104. - Der gegenwärtige Stand und die Bekämpfung der Tuberkulose in Hamburg. Brauers Beitr. Supplementband IV-VIII. 177.

105. - Die Tuberkulosesterblichkeit Hamburgs in den Jahren 1820-1899. Zeitsohr. f. Tuberkul. 1, 1900. Heft 4.

106. Spaeth, Über Verbreitung der Tuberkulose im Kindesalter und deren Bekämpfung. Münch. med. Wochenschr. 1918. Nr. 13.

107. v. Scheibner, Bilden die Tonsillen häufige Eingangspforten für die Tuberkulose? Zieglers Beitr. 26, 511.

108. Schlesinger, Die Krankheiten des höheren Lebensalters. 1914.

109. Schloßmann, Die Tuberkulose als Kinderkrankheit. Münch. med. Wochenschr. 1909. Nr. 8.

110. Schönemann, Arch. f. Laryngol. 22, Nr. 2. Physiologie und Pathologie der Tonsillen.

11. Sokolowski und D mochowski, Ein Beitrag zur Pathologie der entzündlichen Tonsillarprozesse. Deutsch. Arch. f. klin. Med. 49.

112. Staehelin, Über Altersphthise. Berl. klin. Wochenschr. 1910. Nr. 9.

113. Stöhr, Über Mandeln und Balgdrüsen. Virchows Arch. 97.

114. - Über die Mandeln und deren Entwicklung. Korrespondenzbl. f. Schweiz. Ärzte 1890. Nr. 17.

115. Straßmann, Über Tuberkulose der Tonsillen. Virchows Arch. 96.

116. S trü m pell, Lehrb. d. spez. Pathol. u. Therap. 1914.

117. Tachau, Tuberkuloseliteratur im Kriege. Deutsche med. Wochenschr. 1916. Nr. 50.

118. Tendeloo, Pathologische Anatomie der Tuberkulose in Brauers-Schroeders Handbuch der Tuberkulose 1914.

119. - Lymphogene, retrograde Metastasen von Bakterien, Geschwulstzellen und Staub aus der Brust- in die Bauchhöhle, besonders in den paraaortalen Lymphdrüsen. Münch. med. Wochenschr. 1904. Nr. 35.

120. Thiele, Tuberkulöse Kinder. Verlag L. Voß. Leipzig 1915.

121. Umber, Ûber Tuberkuloseinfektion und Tuberkuloseerkrankung der ersten Lebensjahre vor dem Krieg und während desselben. Zeitschr. f. Tuberkul. 27.

122. Wenckebach, Spitzentuberkulose und phthisischer Habitus. Wien. klin. Wochenschr. I918. Nr. 14.

123. Werner, Die Sterblichkeit der Bevölkerung der Bauernschaften Schlangen und Kohlstädt an Tuberkulose von 1901-1909 einschließlich. Brauers Beitr. 24.

124. Westenhoeffer, Über die Wege der tuberkulösen Infektion im kindlichen Körper. Berl. klin. Wochenschr. 1904. Nr. 7.

125. Wood, Bedeutung tuberkulöser Ablagerungen in den Tonsillen. Journ. of Amer. Assoc. 1905. Nr. 18. Ref. Deutsche med. Wochenschr. 1905. Nr. 23. 
Über die neue Tuberkulosewelle; nebst einem Beitrag zum jetzigen Stand usw. 34l

126. Zadeck, Weitere Beiträge zum Verlauf der Lungentuberkulose im Kriege. Münch. med. Wochenschr. 1919. 1194.

127. Ziegler, Infektionswege experimenteller Impftuberkulose, zugleich ein Beitrag zur Lehre von der Lymphbewegung. Med. Klin. 1916.

128. Tuberkulosetodesfätle in Wien. Deutsche med. Wochenschr. 1917. 1240.

129. Die Zunahme der Gesamtmortalität und der Sterblichkeit an Tuberkulose in Deutschland. Intern. Zentralbl. f. d. ges. Tuberkuloseforsch. 1919. Nr. 19.

130. Über die Sterblichkeit an Tuberkulose in Deutschland. „Medizinalstatistische Mitteilungen des Kaiserl. Gesundheitsamtes".

131. Bartschmidt, Über die Einwirkung der Kriegsverhältnisse auf die Tuberkulosehäufigkeit unter den Münchener Kindern. Münch. med. Wochenschr. 1920.

132. Fahr, zur Frage der Kriegswirkung auf Ernährungsverhältnisse. Morbidität und Mortalität. Virch, Arch. 52.

133. Citron, Die Tonsillen als Eingangspforten für Infektionen. Deutsch. med. Wochenschr. 1920. S. 340 . 